\title{
On the three-dimensional Euler equations with a free boundary subject to surface tension
}

\author{
Ben Schweizer \\ Institut für Angewandte Mathematik, Universität Heidelberg, Germany \\ Received 21 July 2004; received in revised form 22 October 2004; accepted 4 November 2004
}

Available online 19 April 2005

\begin{abstract}
We study an incompressible ideal fluid with a free surface that is subject to surface tension; it is not assumed that the fluid is irrotational. We derive a priori estimates for smooth solutions and prove a short-time existence result. The bounds are obtained by combining estimates of energy type with estimates of vorticity type and rely on a careful study of the regularity properties of the pressure function. An adequate artificial coordinate system is used instead of the standard Lagrangian coordinates. Under an assumption on the vorticity, a solution to the Euler equations is obtained as a vanishing viscosity limit of solutions to appropriate Navier-Stokes systems.

(C) 2005 L'Association Publications de l'Institut Henri Poincaré. Published by Elsevier B.V. All rights reserved
\end{abstract}

\section{Introduction}

The investigation of fluids with a free boundary has recently attracted much interest, and numerical studies became possible on the basis of analytical results. Typically, one obtains local existence results that clarify the well-posedness of the equations and identify the adequate function spaces to work with. This was done for the most prominent equations describing incompressible fluids: Navier-Stokes equations, Euler equations for irrotational flow, general Euler equations. In addition, surface tension may be included or neglected.

Depending on the equation, different techniques are used to derive a priori estimates. For the incompressible Navier-Stokes equations with surface tension we mention [1] and [13] for fundamental contributions. Estimates for the solutions can be based on the regularizing effect of the viscosity in this case.

For the Euler equations, no regularizing effect can be exploited in the fluid body; surface tension, if included, has a regularizing effect on the free surface. Existence results for the irrotational Euler equations without surface tension were achieved in [18] and [19]. For results on the irrotational motion of ideal fluids with surface tension see $[2,17]$.

\footnotetext{
E-mail address: ben.schweizer@iwr.uni-heidelberg.de (B. Schweizer).
} 
For the general incompressible Euler equations only partial results are available. For vanishing surface tension, an existence result was obtained in the two-dimensional case in [8]. The three-dimensional case is studied in [4] under a sign condition. The need for such an additional condition is known from [5]. Including surface tension, only the two-dimensional case was treated [11].

Despite the lack of affirmative results, it seems to be a common belief that, even in three dimensions, the regularizing effect of surface tension is strong enough in order to control the nonlinearities of the system (compare [4]). This is confirmed with our Theorem 1, where a priori estimates for solutions are shown. On the other hand, there is not known any iteration procedure which improves approximate solutions in such a way that a fixed point theorem can be applied. In fact, in order to turn our estimates into an existence result, we can not use an iteration, but must approximate the equations by a Navier-Stokes system. Since our estimates are independent of the viscosity we find a local existence result in Theorem 2.

We have to deal with the fact that the Navier-Stokes equations with the natural free boundary conditions will in general not produce approximate solutions to the Euler equation in regular function spaces. We therefore have to impose an artificial boundary condition that relates to the Euler equation. We treat here the case that the vorticity vanishes on the boundary initially (and thus for all times), which provides a tractable condition for the NavierStokes approximation. The condition of vanishing vorticity on the boundary was not needed in the two-dimensional case studied in [11], but a smallness condition for the initial velocity was imposed there. For the analysis of related problems see $[3,9,12]$, and $[7,14]$ for two-phase problems.

Methods in the proof. The fundamental inequality for solutions of the Euler equations is conservation of energy. In the case of a positive surface tension the energy consists of kinetic energy (integral of the squared velocity) and potential energy (proportional to the area or length of the free surface). Energy conservation follows with an integration by parts after testing the equation with the solution. In order to find a priori estimates in spaces of higher regularity (as they are needed for the treatment of the free boundary), it is standard to try the following: One differentiates the equations with respect to time and multiplies with the differentiated solution. The same is done with tangential spatial derivatives. The combination of both should give estimates of energy type for derivatives.

It turns out that this procedure does not work without further estimates in our case. Due to the nonlinearity, the differentiation produces error terms which are of higher order than the positive terms and can therefore not be controlled. The principal idea in this work is to combine the energy estimates with bounds based on the vorticity transport equation. The latter can be used to improve velocity and pressure estimates by half a derivative. Fundamental is the control of highest time derivatives of the pressure in Sobolev spaces of negative order.

The need for the combination of energy and vorticity estimates leads to a difficulty in finding approximate solutions which are needed for the existence result. A discretization of the Euler equations can preserve energy estimates, but we will in general lose the bounds that are based on the vorticity equation. Similarly, an iteration scheme can be constructed to keep the energy bounds, but we did not succeed in keeping additionally the vorticity bounds. Our solution to this problem consist in using the Navier-Stokes equations to construct approximate solutions. The Navier-Stokes system has the same structure as the Euler equations and allows to keep the vorticity estimates.

Equations and main results. We consider the Euler equations in $N$ space dimensions, $N=2$ or $N=3$. We normalize the equations to have all physical constants equal to 1 . They read

$$
\begin{aligned}
& \partial_{t} v+(v \cdot \nabla) v+\nabla p=0, \\
& \operatorname{div} v=0,
\end{aligned}
$$

in the time dependent domain $\Omega_{t}$. We assume that the free boundary is parametrized as the graph of a height function $h$. Then the kinematic boundary condition and the balance of forces (with surface tension) can be written 


$$
\begin{aligned}
& \partial_{t} h-v_{3}+\nabla_{x} h \cdot\left(v_{1}, v_{2}\right)=0, \\
& \nabla_{x} \cdot\left(\frac{\nabla_{x} h}{\sqrt{1+\left|\nabla_{x} h\right|^{2}}}\right)+p=0 .
\end{aligned}
$$

We wrote here the equations for $N=3$. To treat both dimensions simultaneously, we write for $N=2: x$ for $x_{1}, y$ for $x_{2}$, and $\nabla_{x}$ for $\partial_{x_{1}}$. For $N=3: x$ for $\left(x_{1}, x_{2}\right), y$ for $x_{3}$, and $\nabla_{x}$ for $\left(\partial_{x_{1}}, \partial_{x_{2}}\right)$. Later on we will often omit the index of $\nabla_{x}$ when the operator acts on a function of $x$ and $t$. The domain $\Omega_{t}$ is defined by a function $h(x, t)$ as

$$
\Omega_{t}:=\left\{(x, y) \mid x \in[0,1]_{\text {per }}^{N-1},-1<y<h(x, t)\right\},
$$

and the free boundary is

$$
\Gamma_{t}:=\left\{(x, h(x, t)) \mid x \in[0,1]_{\mathrm{per}}^{N-1}\right\} .
$$

We use the flat torus $[0,1]_{\text {per }}^{N-1}$ as horizontal domain, which means that we impose periodicity conditions on the lateral boundaries. We will always write $n=n_{t}$ for the exterior normal of $\Omega_{t}$ on the free boundary, $n(x, h(x, t), t)=$ $(-\nabla h(x, t), 1) / \sqrt{1+|\nabla h(x, t)|^{2}}$. We write $\mathcal{H}^{N-1}$ for the $N-1$-dimensional Hausdorff-measure, in particular, $\mathcal{H}^{N-1}\left(\Gamma_{t}\right)$ is the length of $\Gamma_{t}$ for $N=2$, and the area of the free surface $\Gamma_{t}$ for $N=3$.

A very useful consequence of (1.1) is the transport equation for the vorticity $\omega:=\operatorname{curl} v$, in the case $N=2$ we identify the vorticity with the scalar quantity $\omega=\partial_{2} v_{1}-\partial_{1} v_{2}$.

$$
\begin{array}{ll}
\partial_{t} \omega+(v \cdot \nabla) \omega=0 & \text { for } N=2, \\
\partial_{t} \omega+(v \cdot \nabla) \omega-(\omega \cdot \nabla) v=0 & \text { for } N=3 .
\end{array}
$$

The important observation is that this equation admits regularity estimates of $\omega$. The only restriction to such estimates is the regularity of the normal velocity on the boundary. This connection is made precise in Proposition 3 .

One obtains the (zero-order) energy estimate when testing equation (1.1) with $v$. We find

$$
\partial_{t} \frac{1}{2} \int_{\Omega_{t}}|v|^{2}+\partial_{t} \mathcal{H}^{N-1}\left(\Gamma_{t}\right)=0 .
$$

This equation provides a pointwise (in time) estimate for the kinetic and the potential energy. In a linearized setting, we expect (1.8) to provide bounds for $v \in L^{\infty}\left(0, T ; L^{2}\right), h \in L^{\infty}\left(0, T ; H^{1}\left([0,1]^{N-1}\right)\right)$. Differentiating the equation and testing with derivatives of the solution we expect to find estimates for derivatives of the solution in the same spaces. We call such estimates higher order energy estimates.

In order to define a norm for the function $v$, it is necessary to transform the variable to a reference domain. We parametrize the time dependent domain $\Omega_{t}$ over the rectangle $R:=[0,1]_{\text {per }}^{N-1} \times(-1,0)$ by a map $X(\cdot, t)$,

$$
X(\cdot, t): R \rightarrow \Omega_{t} \text { one-to-one, } \quad X(x, y, t)=(x, Y(x, y, t)) .
$$

We write $S:=[0,1]_{\text {per }}^{N-1} \times\{0\} \equiv[0,1]_{\text {per }}^{N-1}$ for the pre-image of the free surface. To make a choice we set $Y(\cdot, t)$ to be the harmonic function on $R$ with values $h(\cdot, t)$ on $S$, and constant value -1 on $[0,1]_{\text {per }}^{N-1} \times\{-1\}$. We will always be in the case that $h(\cdot, t) \in H^{4}(S, \mathbb{R})$ is small in the Lipschitz norm. In particular, the derivative $\partial_{y} Y$ will be close to 1 pointwise (compare [6], p. 346), and therefore $X(\cdot, t): R \rightarrow \Omega_{t}$ is one-to-one.

We use the following norms for the primary variables.

$$
\begin{aligned}
& v \circ X \in L^{\infty}\left(0, T ; H^{k+3 / 2}(R)\right) \cap \cdots \cap W^{k, \infty}\left(0, T ; H^{3 / 2}(R)\right) \cap W^{k+1, \infty}\left(0, T ; L^{2}(R)\right), \\
& p \circ X \in L^{\infty}\left(0, T ; H^{k+3 / 2}(R)\right) \cap \cdots \cap W^{k-1, \infty}\left(0, T ; H^{2+1 / 2}(R)\right) \cap W^{k, \infty}\left(0, T ; H^{1}(R)\right) \\
& \quad \cap W^{k+1, \infty}\left(0, T ; H_{*}^{-1 / 2}(R)\right), \\
& h \in L^{\infty}\left(0, T ; H^{k+2}(S)\right) \cap \cdots \cap W^{k+1, \infty}\left(0, T ; H^{1}(S)\right) .
\end{aligned}
$$

For a definition of $H_{*}^{-1 / 2}(R)$ see Lemma 7 . 
We next state our first theorem. It provides the a priori bounds for solutions and is proved by combining the energy estimates of Section 2 with the improved velocity and pressure estimates of Proposition 3 . The statement is, loosely speaking, that the solution is as good as the initial values allow. More precisely, the norm of the solution can be estimated by the norm of the initial values, where the appropriate norm for initial values is obtained by taking the infimum of the time-dependent norm over all continuations of the initial values. The precise definition is given in Assumption 5 on page 769 , we abbreviate the norm of initial values $\left(v^{0}, h^{0}\right)$ of Assumption 5 by $\left\|\left(v^{0}, h^{0}\right)\right\|_{\mathrm{IV}}$. In the statements we use $\mathbb{R}_{+}=(0, \infty)$.

Theorem 1. For $k \in \mathbb{N}, k \geqslant 3$, there exist $\eta>0$ and $T_{0} \in C\left(\mathbb{R}_{+}, \mathbb{R}_{+}\right)$such that the following holds. Every solution $(v, p, h)$ of the Euler equations with free boundary $(1.1)-(1.4)$ on $(0, T)$ in the function spaces of (1.10)-(1.12) for index $k+1$ with $\|h(\cdot, 0)\|_{H^{2}(S) \cap C^{0,1}(S)} \leqslant \eta$ and $T \leqslant T_{0}\left(\left\|\left(v^{0}, h^{0}\right)\right\|_{\text {IV }}\right)$ satisfies

$$
\|v\|+\|p\|+\|h\| \leqslant C
$$

in the norms of (1.10)-(1.12). The positive number $C$ depends only on $\left\|\left(v^{0}, h^{0}\right)\right\|_{\mathrm{IV}}$.

Our second theorem provides the existence of solutions. It is proved in Section 4 via uniform (in $v$ ) estimates for a Navier-Stokes system.

Theorem 2. For $k \in \mathbb{N}, k \geqslant 3$, there exists $\eta>0$ such that the following holds. Let $\left(v^{0}, h^{0}\right)$ be $C^{\infty}$ initial values with $\left\|h^{0}\right\|_{H^{2}(S) \cap C^{0,1}(S)} \leqslant \eta$ and with initial vorticity curl $v^{0}$ vanishing on the free boundary $\Gamma_{0}$. Then there exist $T>0$ and $a$ solution of the Euler equations (1.1)-(1.4) on the time interval $(0, T)$.

Moreover, the value $T>0$ depends only on the norms of the initial values as given by Assumption 5. The solution is a vanishing viscosity limit of solutions of a Navier-Stokes approximation.

Remarks on the theorems. (1) On the initial data we only impose smallness of h, i.e. we only demand that the initial parametrization of the domain $\Omega_{0}$ is close to the identity, independent of the velocity. (2) The bounds for the pressure in (1.11) improve the estimates for the height function and provide bounds for

$$
h \in L^{\infty}\left(0, T ; H^{k+3}(S)\right) \cap \cdots \cap W^{k-1, \infty}\left(0, T ; H^{4}(S)\right) \cap W^{k, \infty}\left(0, T ; H^{2+1 / 2}(S)\right) .
$$

(3) The assumption on the initial values to be $C^{\infty}$ in the existence result can be circumvented by an approximation argument, since $T$ depends only on the norms of the initial values.

The key in the proof of both theorems is the following proposition. It states that the variable $h$ (i.e. the evolution of the domain) controls the other primary variables $v$ and $p$. The proof exploits the vorticity equation (1.7) and is presented in Section 3.

Proposition 3. For $k \in \mathbb{N}, k \geqslant 3$, there exist $\eta>0$ and $T_{0} \in C\left(\mathbb{R}_{+}, \mathbb{R}_{+}\right)$such that the following holds. Let $(v, p, h)$ be a smooth solution of the Euler equations on $(0, T)$ with $T \leqslant T_{0}\left(\left\|\left(v^{0}, h^{0}\right)\right\|_{\mathrm{IV}}\right),\|h(\cdot, t)\|_{H^{2}(S) \cap C^{0,1}(S)} \leqslant \eta$ for all $t \in[0, T]$. Then the velocity field $v$ and the pressure $p$ satisfy a bound

$$
\|v\|+\|p\| \leqslant C\left(\|h\|,\left\|\left(v^{0}, h^{0}\right)\right\|_{\mathrm{IV}}\right) .
$$

Here the norm of $v$ is that of (1.10) without the $W^{k+1, \infty}$-estimate. The norm of $p$ is that of (1.11) without the $W^{k, \infty}$-estimate. $C$ depends on the norm of $h$ in (1.12). 


\section{Growth inequalities for regular energies}

Our aim in this section is to derive the energy estimates for derivatives of the physical variables. They include the desired estimates for $h$, but they do not provide sufficient regularity of the velocity. Instead, the $(k+1)$-st order energy estimate refers to the spaces

$$
\begin{aligned}
& v \circ X \in L^{\infty}\left(0, T ; H^{k+1}(R)\right) \cap \cdots \cap W^{k+1, \infty}\left(0, T ; L^{2}(R)\right), \\
& h \in L^{\infty}\left(0, T ; H^{k+2}(S)\right) \cap \cdots \cap W^{k+1, \infty}\left(0, T ; H^{1}(S)\right) .
\end{aligned}
$$

In order to control error terms we must use Proposition 3. It improves the estimates for $v$ by half an order to those of (1.10), and yields bounds for $p$ as in (1.11).

When differentiating the solution we have to perform all derivatives in tangential directions to the free boundary in space and time, either in temporal or in a horizontal direction. This way we can exploit differentiated boundary conditions in the calculations. With the scalar field $Y_{t}:=\partial_{t} Y \circ X^{-1}$ we introduce a vertical material derivative as

$$
\widehat{D}_{t} u(x, y, t):=\partial_{t} u(x, y, t)+\partial_{y} u(x, y, t) Y_{t}(x, y, t) .
$$

This quantity is a total time derivative of $u$ in the moving co-ordinates $X$ in the sense that the following chain-rule holds. The function $u \circ X$ has the time derivative

$$
\partial_{t}(u \circ X)=\frac{\mathrm{d}}{\mathrm{d} t}(u \circ X)=\left(\partial_{t} u\right) \circ X+\left(\nabla_{(x, y)} u\right) \circ X \cdot \partial_{t} X=\left(\partial_{t} u\right) \circ X+\left(\partial_{y} u\right) \circ X \partial_{t} Y=\widehat{D}_{t} u \circ X,
$$

or, equivalently,

$$
\partial_{t}(u \circ X)(x, y, t)=\widehat{D}_{t} u(x, Y(x, y, t), t),
$$

for all $(x, y) \in R$ and all $t$. Note that on the boundary holds the equality

$$
Y_{t}(x, h(x, t), t)=\partial_{t} Y(x, 1, t)=\partial_{t} h(x, t)=v_{n} \sqrt{1+|\nabla h|^{2}} .
$$

We can introduce these derivatives of the solution as new variables. We define

$$
v_{t}:=\widehat{D}_{t} v, \quad p_{t}:=\widehat{D}_{t} p .
$$

We wish to emphasize at this point that we understand $v_{t}$ and $p_{t}$ as new variables - at no point of this article a subscript indicates a derivative. Instead, $Y_{t}, v_{t}, f_{t}, g_{t}, f_{x_{i}}$, and other functions will be new variables, their name is chosen to remind us their definition or their meaning (e.g. $f_{t}$ an error term introduced through time differentiation).

We can also introduce variables corresponding to higher derivatives in the form $\widehat{D}_{t}^{k} v, \widehat{D}_{t}^{k} p$. Moreover, the same idea can also be used for spatial derivatives. We set $Y_{i}:=\partial_{x_{i}} Y \circ X^{-1}$, and introduce a tangential derivative as

$$
\widehat{D}_{x_{i}} u(x, y, t):=\partial_{x_{i}} u(x, y, t)+\partial_{y} u(x, y, t) Y_{i}(x, y, t) .
$$

Note that

$$
Y_{i}(x, h(x, t), t)=\partial_{x_{i}} Y(x, 1, t)=\partial_{x_{i}} h(x, t)
$$

on the boundary. We can also introduce higher derivatives of the form $\widehat{D}_{x_{i}}^{k} v, \widehat{D}_{x_{i}}^{k} p$ as new variables.

The goal of this section is to derive equations for the functions $\left(\widehat{D}_{t}^{k} v, \widehat{D}_{t}^{k} p\right)$ and $\left(\widehat{D}_{x_{i}}^{k} v, \widehat{D}_{x_{i}}^{k} p\right)$. The equations will have the same structure as the initial system. We can therefore repeat the testing procedure applied for (1.8), just that now we will, for example, multiply the equation for $\widehat{D}_{t}^{k} v$ with the function $\widehat{D}_{t}^{k} v$. This will provide the high-order energy estimates of (2.1) and (2.2).

We will make use of the following observation. The testing procedure does not exactly provide the estimates of $h$ as in (2.2), but we will rather find estimates for weighted $L^{2}$-norms of derivatives of $h$, the weight being a function of $\sqrt{1+|\nabla h|^{2}}$ as in e.g. (2.7) or (2.10). Nevertheless, by the assumption in Theorem 1, the bound $|\nabla h| \leqslant \eta$ holds 
initially. As long as $h$ continues to satisfy the bound $|\nabla h| \leqslant 2 \eta$, the weighted energies are equivalent to standard Sobolev norms and we find the $L^{2}$-type estimates. Now the energy-bounds imply that $|\nabla h(\cdot, t)|_{\infty}$ can only grow linearly in time, therefore $|\nabla h| \leqslant 2 \eta$ remains valid on a time interval of a length determined by the initial values. The precise argument is used in the conclusion of the theorem with estimate (2.25).

\subsection{First derivatives in $2 D$}

The vertical material derivative does not commute with standard derivatives. This is the source of the highest order error terms. We have the commutator

$$
\left[\partial_{i}, \widehat{D}_{t}\right] u:=\partial_{i}\left(\partial_{t}+Y_{t} \partial_{y}\right) u-\left(\partial_{t}+Y_{t} \partial_{y}\right) \partial_{i} u=\partial_{i} Y_{t} \partial_{y} u,
$$

and here $i$ can stand for $x_{1}, x_{2}, y$, or $t$. Using the standard abbreviation $\frac{D}{D t}=\partial_{t}+(v \cdot \nabla)$, the equation for $v_{t}=\widehat{D}_{t}^{1} v$ and $p_{t}=\widehat{D}_{t}^{1} p$ reads

$$
\begin{aligned}
\frac{D}{D t} v_{t} & =\partial_{t} \widehat{D}_{t} v+(v \cdot \nabla) \widehat{D}_{t} v \\
& =\widehat{D}_{t} \frac{D}{D t} v+\partial_{t} Y_{t} \partial_{y} v-\left(\widehat{D}_{t} v \cdot \nabla\right) v+(v \cdot \nabla) Y_{t} \partial_{y} v \\
& =-\widehat{D}_{t} \nabla p+\partial_{t} Y_{t} \partial_{y} v-\left(\widehat{D}_{t} v \cdot \nabla\right) v+(v \cdot \nabla) Y_{t} \partial_{y} v \\
& =-\nabla \widehat{D}_{t} p+\nabla Y_{t} \partial_{y} p+\partial_{t} Y_{t} \partial_{y} v-\left(\widehat{D}_{t} v \cdot \nabla\right) v+(v \cdot \nabla) Y_{t} \partial_{y} v \\
& =:-\nabla p_{t}+f_{t}^{1}
\end{aligned}
$$

Testing this equation with $v_{t}$ and using

$$
\operatorname{div} v_{t}=\sum_{j=1}^{N} \partial_{j} Y_{t} \partial_{y} v_{j}=: g_{t}^{1}
$$

we find

$$
\partial_{t} \frac{1}{2} \int_{\Omega_{t}}\left|v_{t}\right|^{2}+\int_{\Gamma_{t}} p_{t} v_{t} \cdot n=\int_{\Omega_{t}} f_{t}^{1} \cdot v_{t}+\int_{\Omega_{t}} g_{t}^{1} p_{t} .
$$

We already see that our choice of coordinates will make it necessary to control highest time derivatives of the pressure. Such an estimate can not be derived from the energy itself.

We next want to identify, up to error terms, the boundary integral on the left-hand side of (2.6) with the time derivative of an energy. We calculate for the first factor

$$
\begin{aligned}
p_{t}(x, h(x, t), t) & =\left(\widehat{D}_{t} p\right)(x, h(x, t), t) \stackrel{(2.3)}{=} \frac{\mathrm{d}}{\mathrm{d} t} p(x, h(x, t), t) \\
& \stackrel{(1.4)}{=}-\partial_{t} \nabla \cdot\left(\frac{\nabla h}{\sqrt{1+|\nabla h|^{2}}}\right) \\
& =-\nabla \cdot\left(\frac{\partial_{t} \nabla h}{\sqrt{1+|\nabla h|^{2}}}-\frac{\nabla h\left\langle\nabla h, \partial_{t} \nabla h\right\rangle}{{\sqrt{1+|\nabla h|^{2}}}^{3}}\right) \\
& \stackrel{(2 \mathrm{D})}{=}-\nabla \cdot\left(\frac{\partial_{t} \nabla h}{\sqrt{1+|\nabla h|^{2}}}\right) .
\end{aligned}
$$

With the notation $\widehat{D}_{t} n=\partial_{t}(n \circ X)$, or, evaluated in a point,

$$
\widehat{D}_{t} n(x, h(x, t), t)=\frac{\mathrm{d}}{\mathrm{d} t}\left[(-\nabla h(x, t), 1) / \sqrt{1+|\nabla h(x, t)|^{2}}\right],
$$


we now calculate for the second factor

$$
\begin{aligned}
v_{t} \cdot n & =\widehat{D}_{t} v \cdot n=\widehat{D}_{t}(v \cdot n)-v \cdot \widehat{D}_{t} n \\
& \stackrel{(1.3)}{=} \partial_{t}\left(\frac{\partial_{t} h}{\sqrt{1+|\nabla h|^{2}}}\right)-v \cdot \widehat{D}_{t} n \\
& =\frac{\partial_{t}^{2} h}{\sqrt{1+|\nabla h|^{2}}}-\frac{\partial_{t} h\left\langle\nabla h, \partial_{t} \nabla h\right\rangle}{\sqrt{1+|\nabla h|^{2}}}-v \cdot \partial_{t}\left(\frac{(-\nabla h, 1)}{\sqrt{1+|\nabla h|^{2}}}\right) \\
& =: \frac{\partial_{t}^{2} h}{\sqrt{1+|\nabla h|^{2}}}+\delta_{t}^{1} .
\end{aligned}
$$

We therefore find for the integral

$$
\begin{aligned}
\int_{\Gamma_{t}} p_{t} v_{t} \cdot n= & \int_{S} p_{t} v_{t} \cdot n \sqrt{1+|\nabla h|^{2}} \\
= & -\int_{S} \nabla \cdot\left(\frac{\partial_{t} \nabla h}{{\sqrt{1+|\nabla h|^{2}}}^{3}}\right)\left(\frac{\partial_{t}^{2} h}{\sqrt{1+|\nabla h|^{2}}}+\delta_{t}^{1}\right) \sqrt{1+|\nabla h|^{2}} \\
= & \int_{S}\left(\frac{\partial_{t} \nabla h}{\sqrt{1+|\nabla h|^{2}}}\right) \cdot \nabla\left(\partial_{t}^{2} h+\delta_{t}^{1} \sqrt{1+|\nabla h|^{2}}\right) \\
= & \partial_{t} \frac{1}{2} \int_{S} \frac{\left|\partial_{t} \nabla h\right|^{2}}{{\sqrt{1+|\nabla h|^{2}}}^{3}}+\frac{3}{2} \int_{S}\left|\partial_{t} \nabla h\right|^{2} \frac{\left\langle\nabla h, \partial_{t} \nabla h\right\rangle}{\sqrt{1+|\nabla h|^{2}}} \\
& +\int_{S}\left(\frac{\partial_{t} \nabla h}{{\sqrt{1+|\nabla h|^{2}}}^{3}}\right) \cdot \nabla\left(\delta_{t}^{1} \sqrt{1+|\nabla h|^{2}}\right) .
\end{aligned}
$$

For the integrand of the second integral we introduce the abbreviation

$$
\varepsilon_{t}^{1}:=-\frac{3}{2}\left|\partial_{t} \nabla h\right|^{2} \frac{\left\langle\nabla h, \partial_{t} \nabla h\right\rangle}{{\sqrt{1+|\nabla h|^{2}}}^{5}} .
$$

We can now introduce the first order energy

$$
E_{t}^{1}(v, h):=\frac{1}{2} \int_{\Omega_{t}}\left|v_{t}\right|^{2}+\frac{1}{2} \int_{S} \frac{\left|\partial_{t} \nabla h\right|^{2}}{{\sqrt{1+|\nabla h|^{2}}}^{3}},
$$

and estimate (2.6) can be written as

$$
\partial_{t} E_{t}^{1}=\int_{\Omega_{t}} f_{t}^{1} \cdot v_{t}+\int_{\Omega_{t}} g_{t}^{1} p_{t}+\int_{S} \varepsilon_{t}^{1}-\int_{S}\left(\frac{\partial_{t} \nabla h}{\sqrt{1+|\nabla h|^{2}}}\right) \cdot \nabla\left(\delta_{t}^{1} \sqrt{1+|\nabla h|^{2}}\right) .
$$

We will see that all terms on the right-hand side can be treated as error terms. Our next aim must therefore be to analyze the quality of the integrals on the right-hand side. To this end we use jets: Given a $H^{k}$ function $u=$ $u(x, y, t)$, the symbol $\bar{D}^{k} u$ denotes the vector of all derivatives of $u$ up to order $k$. The symbol $\bar{\partial}^{k} u$ is used for the jet of functions $u=u(x, t)$. By $\mathcal{P}\left(\xi_{1}, \ldots, \xi_{n}\right)$ we denote the class of polynomials in the variables $\xi_{1}, \ldots, \xi_{n}$, and additionally in ${\sqrt{1+|\nabla h|^{2}}}^{ \pm 1}$. We inspect the error terms and find

$$
f_{t}^{1} \in \mathcal{P}\left(\bar{D}^{1} v, \bar{D}^{1} Y_{t}, \nabla p\right), \quad g_{t}^{1} \in \mathcal{P}\left(\nabla v, \nabla Y_{t}\right), \quad \delta_{t}^{1} \in \mathcal{P}\left(\bar{\partial}^{2} h, v\right), \quad \varepsilon_{t}^{1} \in \mathcal{P}\left(\bar{\partial}^{2} h\right) .
$$


The calculations leading to (2.8) can be repeated for the tangential derivatives $\widehat{D}_{x_{i}} v$ and $\widehat{D}_{x_{i}} p$. We exploit that the derivative has a tangential direction when calculating

$$
\left(\widehat{D}_{x_{i}} p\right)(x, h(x, t), t)=\frac{\mathrm{d}}{\mathrm{d} x_{i}} p(x, h(x, t), t) .
$$

Introducing the energy

$$
E_{x_{i}}^{1}(v, h):=\frac{1}{2} \int_{\Omega_{t}}\left|\widehat{D}_{x_{i}} v\right|^{2}+\frac{1}{2} \int_{S} \frac{\left|\partial_{x_{i}} \nabla h\right|^{2}}{{\sqrt{1+|\nabla h|^{2}}}^{3}},
$$

we find the estimate

$$
\partial_{t} E_{x_{i}}^{1}=\int_{\Omega_{t}} f_{x_{i}}^{1} \cdot v_{x_{i}}+\int_{\Omega_{t}} g_{x_{i}}^{1} p_{x_{i}}+\int_{S} \varepsilon_{x_{i}}^{1}-\int_{S}\left(\frac{\partial_{x_{i}} \nabla h}{\sqrt{1+|\nabla h|^{2}}}\right) \cdot \nabla\left(\delta_{x_{i}}^{1} \sqrt{1+|\nabla h|^{2}}\right) .
$$

Here the error terms $f_{x_{i}}^{1}, g_{x_{i}}^{1}, \delta_{x_{i}}^{1}$, and $\varepsilon_{x_{i}}^{1}$ have the structure (2.9) just as their temporal counterparts.

The sum of the two energies (three for $N=3), E_{t}^{1}+\sum_{i} E_{x_{i}}^{1}$, can yield bounds for $h \in L^{\infty}\left(0, T ; H^{2}(S)\right) \cap$ $W^{1, \infty}\left(0, T ; H^{1}(S)\right)$. This coincides with (2.2) for $k=0$. On this basis Proposition 3 can provide bounds for $v$ and $p$. Once the error integrals can be estimated by these norms, (2.8) and (2.11) yield the desired a priori estimate.

\subsection{Higher derivatives in $2 D$}

In this subsection we use an inductive procedure to calculate the equations and the estimates for higher derivatives. We start from the equation on level $k$,

$$
\frac{D}{D t} \widehat{D}_{t}^{k} v+\nabla \widehat{D}_{t}^{k} p=f_{t}^{k}
$$

as it was derived for $k=1$ in 2.1. A further differentiation of $v$ yields the same equation for $\widehat{D}_{t}^{k+1} v$ and the expression for $f_{t}^{k+1}$.

$$
\begin{aligned}
\frac{D}{D t} \widehat{D}_{t}^{k+1} v & =\partial_{t} \widehat{D}_{t} \widehat{D}_{t}^{k} v+(v \cdot \nabla) \widehat{D}_{t} \widehat{D}_{t}^{k} v \\
& =\widehat{D}_{t} \partial_{t} \widehat{D}_{t}^{k} v+\partial_{t} Y_{t} \partial_{y} \widehat{D}_{t}^{k} v+\widehat{D}_{t}\left[(v \cdot \nabla) \widehat{D}_{t}^{k} v\right]-\left(\widehat{D}_{t} v \cdot \nabla\right) \widehat{D}_{t}^{k} v+(v \cdot \nabla) Y_{t} \partial_{y} \widehat{D}_{t}^{k} v \\
& =\widehat{D}_{t} \frac{D}{D t} \widehat{D}_{t}^{k} v+\partial_{t} Y_{t} \partial_{y} \widehat{D}_{t}^{k} v-\left(\widehat{D}_{t} v \cdot \nabla\right) \widehat{D}_{t}^{k} v+(v \cdot \nabla) Y_{t} \partial_{y} \widehat{D}_{t}^{k} v \\
& \stackrel{(2.12)}{=} \widehat{D}_{t} \nabla \widehat{D}_{t}^{k} p+\widehat{D}_{t} f_{t}^{k}+\partial_{t} Y_{t} \partial_{y} \widehat{D}_{t}^{k} v-\left(\widehat{D}_{t} v \cdot \nabla\right) \widehat{D}_{t}^{k} v+(v \cdot \nabla) Y_{t} \partial_{y} \widehat{D}_{t}^{k} v \\
& =-\nabla \widehat{D}_{t} \widehat{D}_{t}^{k} p+\widehat{D}_{t} f_{t}^{k}+\nabla Y_{t} \partial_{y} \widehat{D}_{t}^{k} p+\partial_{t} Y_{t} \partial_{y} \widehat{D}_{t}^{k} v-\left(\widehat{D}_{t} v \cdot \nabla\right) \widehat{D}_{t}^{k} v+(v \cdot \nabla) Y_{t} \partial_{y} \widehat{D}_{t}^{k} v \\
& =:-\nabla \widehat{D}_{t}^{k+1} p+f_{t}^{k+1} .
\end{aligned}
$$

By induction we can conclude for the structure of $f_{t}^{k+1}$

$$
\begin{array}{ll}
f_{t}^{k+1} \in \mathcal{P}^{(1)}\left(\bar{D}^{k+1} v, \bar{D}^{k+1} Y_{t}, \bar{D}^{k} \nabla p\right) & \forall k \geqslant 1, \\
f_{t}^{k+1} \in \mathcal{P}^{(1,1)}\left(\bar{D}^{k+1} v, \bar{D}^{k+1} Y_{t}, \bar{D}^{k} \nabla p\right) & \forall k \geqslant 3 .
\end{array}
$$

Here we use the following short notation for polynomials: $\mathcal{P}^{(1)}$ is the class of all polynomials that are affine in all entries that represent highest derivatives. $\mathcal{P}^{(1,1)}$ denotes polynomials that are affine in all entries that represent highest or next to highest derivatives.

Concerning the divergence we start from the equation

$$
\operatorname{div} \widehat{D}_{t}^{k} v=g_{t}^{k} \text {. }
$$


Again, $g_{t}^{k}$ was calculated for $k=1$ in the last subsection. We derive

$$
\operatorname{div} \widehat{D}_{t}^{k+1} v=\widehat{D}_{t} \operatorname{div} \widehat{D}_{t}^{k} v+\sum_{j} \partial_{j} Y_{t} \partial_{y} \widehat{D}_{t}^{k} v_{j}=\widehat{D}_{t} g_{t}^{k}+\sum_{j} \partial_{j} Y_{t} \partial_{y} \widehat{D}_{t}^{k} v_{j}=: g_{t}^{k+1}
$$

By induction follows

$$
\begin{array}{ll}
g_{t}^{k+1} \in \mathcal{P}^{(1)}\left(\bar{D}^{k} \nabla v, \bar{D}^{k} \nabla Y_{t}\right) & \forall k \geqslant 1, \\
g_{t}^{k+1} \in \mathcal{P}^{(1,1)}\left(\bar{D}^{k} \nabla v, \bar{D}^{k} \nabla Y_{t}\right) & \forall k \geqslant 3 .
\end{array}
$$

We now test (2.12) on level $k+1$ with $\widehat{D}_{t}^{k+1} v$ and find

$$
\partial_{t} \frac{1}{2} \int_{\Omega_{t}}\left|\widehat{D}_{t}^{k+1} v\right|^{2}+\int_{\Gamma_{t}} \widehat{D}_{t}^{k+1} p \widehat{D}_{t}^{k+1} v \cdot n=\int_{\Omega_{t}} f_{t}^{k+1} \cdot \widehat{D}_{t}^{k+1} v+\int_{\Omega_{t}} g_{t}^{k+1} \widehat{D}_{t}^{k+1} p .
$$

We again want to identify the boundary integral on the left-hand side as the time derivative of a positive function. Starting from

$$
\left(\widehat{D}_{t}^{k} p\right)(x, h(x, t), t) \stackrel{(2 \mathrm{D})}{=}-\nabla \cdot\left(\frac{\partial_{t}^{k} \nabla h}{{\sqrt{1+|\nabla h|^{2}}}^{3}}\right)+\rho_{t}^{k},
$$

where $\rho_{t}^{k}=\rho_{t}^{k}(x, t)$ is considered as a function of $x$ and $t$, we derive the expression for $\rho_{t}^{k+1}$ as

$$
\begin{aligned}
& \widehat{D}_{t}^{k+1} p(x, h(x, t), t)=\frac{\mathrm{d}}{\mathrm{d} t}\left[\left(\widehat{D}_{t}^{k} p\right)(x, h(x, t), t)\right] \\
& \stackrel{(2.15)}{=}-\partial_{t} \nabla \cdot\left(\frac{\partial_{t}^{k} \nabla h}{{\sqrt{1+|\nabla h|^{2}}}^{3}}\right)+\partial_{t} \rho_{t}^{k} \\
&=-\nabla \cdot\left(\frac{\partial_{t}^{k+1} \nabla h}{{\sqrt{1+|\nabla h|^{2}}}^{3}}-3 \frac{\partial_{t}^{k} \nabla h\left\langle\nabla h, \partial_{t} \nabla h\right\rangle}{{\sqrt{1+|\nabla h|^{2}}}^{5}}\right)+\partial_{t} \rho_{t}^{k} \\
&=:-\nabla \cdot\left(\frac{\partial_{t}^{k+1} \nabla h}{{\sqrt{1+|\nabla h|^{2}}}^{3}}\right)+\rho_{t}^{k+1} .
\end{aligned}
$$

Starting induction with $\rho_{t}^{1}=0$ we find

$$
\begin{array}{ll}
\rho_{t}^{k+1} \in \mathcal{P}^{(1)}\left(\bar{\partial}^{k} \nabla^{2} h, \bar{\partial}^{k} \nabla h, \bar{\partial}^{k} h\right) & \forall k \geqslant 1, \\
\rho_{t}^{k+1} \in \mathcal{P}^{(1,1)}\left(\bar{\partial}^{k} \nabla^{2} h, \bar{\partial}^{k} \nabla h, \bar{\partial}^{k} h\right) & \forall k \geqslant 3 .
\end{array}
$$

We finally calculate the velocity, starting from equation

$$
\widehat{D}_{t}^{k} v \cdot n=\frac{\partial_{t}^{k+1} h}{\sqrt{1+|\nabla h|^{2}}}+\delta_{t}^{k}
$$

on the boundary. The derivative $\widehat{D}_{t}$ uses only the boundary values and we calculate

$$
\begin{aligned}
\widehat{D}_{t}^{k+1} v \cdot n & =\widehat{D}_{t}\left(\widehat{D}_{t}^{k} v\right) \cdot n=\widehat{D}_{t}\left(\widehat{D}_{t}^{k} v \cdot n\right)-\widehat{D}_{t}^{k} v \cdot \widehat{D}_{t} n=\partial_{t}\left(\frac{\partial_{t}^{k+1} h}{\sqrt{1+|\nabla h|^{2}}}+\delta_{t}^{k}\right)-\widehat{D}_{t}^{k} v \cdot \widehat{D}_{t} n \\
& =\frac{\partial_{t}^{k+2} h}{\sqrt{1+|\nabla h|^{2}}}-\frac{\partial_{t}^{k+1} h\left\langle\nabla h, \partial_{t} \nabla h\right\rangle}{{\sqrt{1+|\nabla h|^{2}}}^{3}}+\partial_{t} \delta_{t}^{k}-\widehat{D}_{t}^{k} v \cdot \partial_{t}\left(\frac{(-\nabla h, 1)}{\sqrt{1+|\nabla h|^{2}}}\right) \\
& =: \frac{\partial_{t}^{k+2} h}{\sqrt{1+|\nabla h|^{2}}}+\delta_{t}^{k+1} .
\end{aligned}
$$


In the analysis of error terms introduced by $\delta_{t}^{k}$ some care is required. The expression $\partial_{t}^{k} \delta_{t}^{1}$ appears in $\delta_{t}^{k+1}$. This is the only term of order $k+2$ in $h$. We recall

$$
\delta_{t}^{1}=F \cdot \partial_{t} \nabla h \quad \text { with } F \in \mathcal{P}\left(\bar{\partial}^{1} h, v\right) .
$$

For the other contributions we find

$$
\begin{array}{ll}
\delta_{t}^{k+1}-\partial_{t}^{k} \delta_{t}^{1} \in \mathcal{P}^{(1)}\left(\bar{\partial}^{k+1} h, \bar{D}^{k} v\right) & \forall k \geqslant 2, \\
\delta_{t}^{k+1}-\partial_{t}^{k} \delta_{t}^{1} \in \mathcal{P}^{(1,1)}\left(\bar{\partial}^{k+1} h, \bar{D}^{k} v\right) & \forall k \geqslant 3 .
\end{array}
$$

Concerning the second entry we emphazise that the polynomial is expressed in terms of $\bar{D}^{k} v(x, h(x, t), t)$.

We are now in a position to derive energy estimates in $k$-th order. We introduce the abbreviation

$$
\varepsilon_{t}^{k+1}:=-\frac{3}{2}\left|\partial_{t}^{k+1} \nabla h\right|^{2} \frac{\nabla h \cdot \partial_{t} \nabla h}{\sqrt{1+|\nabla h|^{2}}}
$$

and note that for $k \geqslant 2$

$$
\varepsilon_{t}^{k+1} \in \mathcal{P}^{(2,0)}\left(\bar{\partial}^{k+1} \nabla h\right),
$$

where $\mathcal{P}^{(2,0)}$ denotes the class of polynomials that are quadratic in the entries for highest derivatives and are independent of entries for next to highest derivatives. Combining the above equalities we get

$$
\begin{aligned}
\int_{\Gamma} & \widehat{D}_{t}^{k+1} p \widehat{D}_{t}^{k+1} v \cdot n=\int_{S} \widehat{D}_{t}^{k+1} p \widehat{D}_{t}^{k+1} v \cdot n \sqrt{1+|\nabla h|^{2}} \\
= & \int_{S}\left[-\nabla \cdot\left(\frac{\partial_{t}^{k+1} \nabla h}{\sqrt{1+|\nabla h|^{2}}}\right)+\rho_{t}^{k+1}\right]\left(\frac{\partial_{t}^{k+2} h}{\sqrt{1+|\nabla h|^{2}}}+\delta_{t}^{k+1}\right) \sqrt{1+|\nabla h|^{2}} \\
= & \int_{S}\left(\frac{\partial_{t}^{k+1} \nabla h}{\sqrt{1+|\nabla h|^{2}}}\right) \cdot \nabla\left(\partial_{t}^{k+2} h+\delta_{t}^{k+1} \sqrt{1+|\nabla h|^{2}}\right)+\int_{S} \rho_{t}^{k+1}\left(\partial_{t}^{k+2} h+\delta_{t}^{k+1} \sqrt{1+|\nabla h|^{2}}\right) \\
= & \partial_{t} \frac{1}{2} \int_{S} \frac{\left|\partial_{t}^{k+1} \nabla h\right|^{2}}{{\sqrt{1+|\nabla h|^{2}}}^{3}}-\int_{S} \varepsilon_{t}^{k+1}+\int_{S}\left(\frac{\partial_{t}^{k+1} \nabla h}{\sqrt{1+|\nabla h|^{2}}}\right) \cdot \nabla\left(\delta_{t}^{k+1} \sqrt{1+|\nabla h|^{2}}\right) \\
& +\int_{S} \rho_{t}^{k+1}\left(\partial_{t}^{k+2} h+\delta_{t}^{k+1} \sqrt{1+|\nabla h|^{2}}\right) .
\end{aligned}
$$

For the high-regularity energy

$$
E_{t}^{k+1}(v, h):=\frac{1}{2} \int_{\Omega_{t}}\left|\widehat{D}_{t}^{k+1} v\right|^{2}+\frac{1}{2} \int_{S} \frac{\left|\partial_{t}^{k+1} \nabla h\right|^{2}}{\sqrt{1+|\nabla h|^{2}}}
$$

the estimate (2.14) reads now

$$
\begin{aligned}
\partial_{t} E_{t}^{k+1}= & \int_{\Omega_{t}} f_{t}^{k+1} \cdot \widehat{D}_{t}^{k+1} v+\int_{\Omega_{t}} g_{t}^{k+1} \widehat{D}_{t}^{k+1} p+\int_{S} \varepsilon_{t}^{k+1}-\int_{S}\left(\frac{\partial_{t}^{k+1} \nabla h}{{\sqrt{1+|\nabla h|^{2}}}^{3}}\right) \cdot \nabla\left(\delta_{t}^{k+1} \sqrt{1+|\nabla h|^{2}}\right) \\
& -\int_{S} \rho_{t}^{k+1}\left(\partial_{t}^{k+2} h+\delta_{t}^{k+1} \sqrt{1+|\nabla h|^{2}}\right) .
\end{aligned}
$$


Without repeating the calculations we note that the same inequality holds for tangential derivatives. For the energy

$$
E_{x_{i}}^{k+1}(v, h):=\frac{1}{2} \int_{\Omega_{t}}\left|\widehat{D}_{x_{i}}^{k+1} v\right|^{2}+\frac{1}{2} \int_{S} \frac{\left|\partial_{x_{i}}^{k+1} \nabla h\right|^{2}}{\sqrt{1+|\nabla h|^{2}}}
$$

holds

$$
\begin{aligned}
\partial_{t} E_{x_{i}}^{k+1}= & \int_{\Omega_{t}} f_{x_{i}}^{k+1} \cdot \widehat{D}_{x_{i}}^{k+1} v+\int_{\Omega_{t}} g_{x_{i}}^{k+1} \widehat{D}_{x_{i}}^{k+1} p+\int_{S} \varepsilon_{x_{i}}^{k+1}-\int_{S}\left(\frac{\partial_{x_{i}}^{k+1} \nabla h}{\sqrt{1+|\nabla h|^{2}}}\right) \cdot \nabla\left(\delta_{x_{i}}^{k+1} \sqrt{1+|\nabla h|^{2}}\right) \\
& -\int_{S} \rho_{x_{i}}^{k+1}\left(\partial_{x_{i}}^{k+2} h+\delta_{x_{i}}^{k+1} \sqrt{1+|\nabla h|^{2}}\right) .
\end{aligned}
$$

For $k \geqslant 3$ the error terms satisfy

$$
\begin{aligned}
f_{x_{i}}^{k+1} & \in \mathcal{P}^{(1,1)}\left(\bar{D}^{k+1} v, \bar{D}^{k+1} Y_{i}, \bar{D}^{k} \nabla p\right), \\
g_{x_{i}}^{k+1} & \in \mathcal{P}^{(1,1)}\left(\bar{D}^{k} \nabla v, \bar{D}^{k} \nabla Y_{i}\right), \\
\rho_{x_{i}}^{k+1} & \in \mathcal{P}^{(1,1)}\left(\bar{\partial}^{k} \nabla^{2} h, \bar{\partial}^{k} \nabla h, \bar{\partial}^{k} h\right), \\
\delta_{x_{i}}^{k+1}-\partial_{x_{i}}^{k} \delta_{x_{i}}^{1} & \in \mathcal{P}^{(1,1)}\left(\bar{\partial}^{k+1} h, \bar{D}^{k} v\right), \\
\varepsilon_{x_{i}}^{k+1} & \in \mathcal{P}^{(2,0)}\left(\bar{\partial}^{k+1} \nabla h\right),
\end{aligned}
$$

with

$$
\delta_{x_{i}}^{1}=F_{i} \cdot \partial_{x_{i}} \nabla h \quad \text { for } F_{i} \in \mathcal{P}\left(\bar{\partial}^{1} h, v\right) .
$$

\subsection{Analysis of error terms}

From now on we always assume $k \geqslant 3$. We will use the sum of all the higher energies as a total energy,

$$
E^{k+1}(t):=E_{t}^{k+1}(t)+\cdots+E_{x_{1}}^{k+1}(t)+E_{x_{2}}^{k+1}(t) .
$$

The dots indicate that also energies of mixed derivatives (spatial and temporal) are used. A $t$-independent estimate for the energy $E^{k+1}(t)$ yields immediately a bound for the function $h$,

$$
\|h\|_{L^{\infty}\left(0, T ; H^{k+2}(S)\right)}+\cdots+\|h\|_{W^{k+1, \infty}\left(0, T ; H^{1}(S)\right)} \leqslant C \sup _{\tau \in[0, T]} E^{k+1}(\tau),
$$

where we assume that $|\nabla h(\cdot, t)|_{L^{\infty}} \leqslant 2 \eta$ remains satisfied for all $t \in[0, T]$. Based on this estimate, Proposition 3 yields the estimate

$$
\|v \circ X\|_{L^{\infty}\left(0, T ; H^{k+3 / 2}(R)\right)}+\cdots+\|v \circ X\|_{W^{k, \infty}\left(0, T ; H^{3 / 2}(R)\right)}+\|v \circ X\|_{W^{k+1, \infty}\left(0, T ; L^{2}(R)\right)} \leqslant C_{v},
$$

where $C_{v}$ depends only on $\sup _{\tau \in[0, T]} E^{k+1}(\tau)$. Note that the $W^{k+1, \infty}$ estimate is not taken from the proposition, but directly from the energy $E_{t}^{k+1}$. The corresponding regularity of the pressure is

$$
\begin{aligned}
& \|p \circ X\|_{L^{\infty}\left(0, T ; H^{k+3 / 2}(R)\right)}+\cdots+\|p \circ X\|_{W^{k-1, \infty}\left(0, T ; H^{2+1 / 2}(R)\right)} \\
& \quad+\|p \circ X\|_{W^{k, \infty}\left(0, T ; H^{1}(R)\right)}+\|p \circ X\|_{W^{k+1, \infty}\left(0, T ; H_{*}^{-1 / 2}(R)\right)} \leqslant C_{p},
\end{aligned}
$$


where $C_{p}$ depends only on $\sup _{\tau \in[0, T]} E^{k+1}(\tau)$. Here the $W^{k, \infty}$ estimate is not taken from the proposition; instead we use Eq. (1.1) for $\nabla p$ and conclude from the estimate for $v$. We emphasize at this point that we will use in the following only the estimate

$$
\bar{D}^{k+1}(p \circ X) \in L^{\infty}\left(0, T ; H_{*}^{-1 / 2}(R)\right) .
$$

The final a priori estimate follows now by using the estimates (2.22) and (2.23) in the energy growth inequalities (2.18) and (2.20). It suffices to check that for bounded $v, p$, and $h$, time integrals of the right-hand side in (2.18) are bounded by a small number for $T>0$ small.

For the two integrals

$$
\int_{\Omega_{t}} f_{t}^{k+1} \cdot \widehat{D}_{t}^{k+1} v \text { and } \int_{S} \varepsilon_{t}^{k+1}
$$

the bound is immediate; the integrands are products of two $L^{2}$-functions with other $L^{\infty}$-functions; the norms of all factors are bounded by the energies.

The term

$$
I_{p}:=\int_{0}^{T} \int_{\Omega_{t}} g_{t}^{k+1} \widehat{D}_{t}^{k+1} p
$$

poses a severe problem if one has only energy estimates for $v$ and $p$. Our improved estimates imply that

$$
g_{t}^{k+1} \circ X \sim \bar{D}^{k} \nabla(v \circ X) \in L^{\infty}\left(0, T ; H^{1 / 2}(R)\right),
$$

and $\partial_{t}^{k+1}(p \circ X) \in L^{\infty}\left(0, T ; H_{*}^{-1 / 2}(R)\right)$, that is,

$$
\widehat{D}_{t}^{k+1} p \circ X=Q_{0}+\sum_{j=1}^{N-1} \partial_{x_{j}} Q_{j} \quad \text { with } Q_{j} \in L^{\infty}\left(0, T ; H^{1 / 2}(R)\right) \text {. }
$$

The pairing

$$
H^{1 / 2}(R) \times H^{1 / 2}(R) \ni(g, Q) \mapsto \int_{R} g \partial_{x_{j}} Q
$$

is a bounded map. This implies that $I_{p}$ is small for bounded energies and $T>0$ small.

Other critical terms in the estimate are

$$
\begin{aligned}
I_{1} & :=\int_{0}^{T} \int_{S} \rho_{t}^{k+1} \partial_{t}^{k+2} h, \\
I_{2} & :=\int_{0}^{T} \int_{S}\left(\frac{\partial_{t}^{k+1} \nabla h}{{\sqrt{1+|\nabla h|^{2}}}^{3}}\right) \cdot \nabla\left(\delta_{t}^{k+1} \sqrt{1+|\nabla h|^{2}}\right) .
\end{aligned}
$$

In the analysis of these terms we have to represent the highest order expressions as derivatives. With polynomials $P_{i j} \in \mathcal{P}\left(\bar{\partial}^{k-1} h\right)$ we write the highest order terms of $\rho_{t}^{k+1}$ as $\partial_{x_{i}} \partial_{x_{j}} \partial_{t}^{k} h P_{i j}\left(\bar{\partial}^{k-1} h\right)$. We calculate for the integral

$$
I_{1}^{\prime}=\int_{0}^{T} \int_{S} \partial_{x_{i}} \partial_{x_{j}} \partial_{t}^{k} h P_{i j}\left(\bar{\partial}^{k-1} h\right) \partial_{t}^{k+2} h
$$




$$
\begin{aligned}
= & \int_{0}^{T} \int_{S} \partial_{t}\left[\partial_{x_{i}} \partial_{x_{j}} \partial_{t}^{k} h P_{i j}\left(\bar{\partial}^{k-1} h\right) \partial_{t}^{k+1} h\right]-\int_{0}^{T} \int_{S} \partial_{t}\left[\partial_{x_{i}} \partial_{x_{j}} \partial_{t}^{k} h P_{i j}\left(\bar{\partial}^{k-1} h\right)\right] \partial_{t}^{k+1} h \\
= & -\left.\int_{S} \partial_{x_{j}} \partial_{t}^{k} h \partial_{x_{i}}\left[P_{i j}\left(\bar{\partial}^{k-1} h\right) \partial_{t}^{k+1} h\right]\right|_{0} ^{T}+\iint_{0}^{T} \partial_{S}\left[\partial_{x_{j}} \partial_{t}^{k} h \partial_{x_{i}} P_{i j}\left(\bar{\partial}^{k-1} h\right)\right] \partial_{t}^{k+1} h \\
& +\int_{0}^{T} \int_{S} \partial_{t}\left[\partial_{x_{j}} \partial_{t}^{k} h P_{i j}\left(\bar{\partial}^{k-1} h\right)\right] \partial_{x_{i}} \partial_{t}^{k+1} h .
\end{aligned}
$$

Concerning the first integral we use that $\left\|\partial_{x_{i}} \partial_{t}^{k+1} h(T)\right\|_{L^{2}}^{2}$ is bounded by the energy. The other factor has a time derivative in $L^{\infty}\left(0, T ; L^{2}\right)$ bounded by the energy. We find

$$
I_{1}^{\prime} \leqslant C_{1}\left(1+\left|\sup _{\tau} E^{k+1}(\tau)\right|^{1 / 2}\right)+C_{2} T\left(1+\left|\sup _{\tau} E^{k+1}(\tau)\right|^{m}\right)
$$

with $C_{1}$ and $C_{2}$ depending only on the initial values and $m>0$. Note that also the second and third integral satisfy the bound.

For the integral $I_{2}$ we must exploit that the highest order terms are divergences. $\delta_{t}^{1}$ is a multiple of $\partial_{t} \nabla h$, therefore the highest order terms are

$$
\begin{aligned}
I_{2}^{\prime} & =\int_{0}^{T} \int_{S} \partial_{t}^{k+1} \nabla h P_{i}\left(\bar{\partial}^{k-1} h\right) \partial_{t}^{k+1} \partial_{x_{i}} \nabla h=\int_{0}^{T} \int_{S} P_{i}\left(\bar{\partial}^{k-1} h\right) \partial_{x_{i}}\left(\frac{1}{2}\left|\partial_{t}^{k+1} \nabla h\right|^{2}\right) \\
& =-\int_{0}^{T} \int_{S} \partial_{x_{i}} P_{i}\left(\bar{\partial}^{k-1} h\right) \frac{1}{2}\left|\partial_{t}^{k+1} \nabla h\right|^{2} .
\end{aligned}
$$

This integral again satisfies (2.24).

A critical term of lower order is generated by time derivatives of $v$ contained in $\delta_{t}^{k+1}$. Such terms read

$$
\int_{0}^{T} \int_{S} \partial_{t}^{k+1} \nabla h P\left(\bar{\partial}^{k-1} h\right) \cdot \nabla \widehat{D}_{t}^{k} v .
$$

For this integral we use the estimate for boundary values of $\nabla \widehat{D}_{t}^{k} v$ expressed in (3.6) and can estimate as in (2.24).

Conclusion of Theorem 1 in the case $N=2$. Estimate (2.18) together with (2.20) and their counterparts for mixed derivatives allow now to conclude the proof of Theorem 1. Since the energy estimates imply the $L^{2}$-type estimates of (2.22) and (2.23) only for uniformly bounded $|\nabla h|$, we set $T_{\eta}:=\sup \left\{t \in[0, T]:|\nabla h(\cdot, \tau)|_{\infty} \leqslant 2 \eta \forall \tau \leqslant t\right\}$.

As a preparation we observe that the $L^{\infty}$-bound of $\partial_{t} E^{k+1}(t)$ implies the continuity of the energy on [0, $\left.T_{\eta}\right]$. We now estimate the energy by its initial values and the supremum of its time derivatives and find

$$
\sup _{\tau \in[0, t]} E^{k+1}(\tau) \leqslant C+t \Phi\left(\sup _{\tau \in[0, t]} E^{k+1}(\tau)\right)
$$

for all $t \in\left[0, T_{\eta}\right]$, for a constant $C$ and a polynomial $\Phi$ depending only on the norms of the initial values. Here we absorbed the factor $C_{1}\left|\sup _{\tau \in[0, T]} E^{k+1}(\tau)\right|^{1 / 2}$ of (2.24) in the left-hand side. For $t<T_{0}, T_{0}>0$ small (depending 
on the initial values through $C$ and $\Phi)$, the supremum of the energies can only be either smaller than $C+1$ or larger than $C+2$. Continuity of the energy then implies the uniform bound

$$
\sup _{\tau \in[0, t]} E^{k+1}(\tau) \leqslant C+1
$$

for all $t \in\left[0, \min \left\{T_{\eta}, T_{0}\right\}\right]$. In order to conclude the uniform estimate on a time interval that depends only on the norms of the initial values, it remains to estimate $T_{\eta}$ from below. The bound $\left\|\partial_{t} \nabla h\right\|_{\infty} \leqslant C$ on $\left[0, \min \left\{T_{\eta}, T_{0}\right\}\right]$ implies $\|\nabla h(\cdot, t)\|_{\infty} \leqslant \eta+C t$ for $t \in\left[0, \min \left\{T_{\eta}, T_{0}\right\}\right]$, and hence $T_{\eta} \geqslant \min \left\{\eta / C, T_{0}\right\}$. In particular, the value of $\min \left\{T_{\eta}, T_{0}\right\}$ depends only on the norm of the initial values.

\subsection{Estimates in the three-dimensional case}

In our approach there is not much difference between the cases $N=2$ and $N=3$. In the two-dimensional case the expression for $p_{t}$ on page simplifies in the last line. This lead to $\rho_{t}^{1}=0$ in Eq. (2.15). In the three-dimensional case the equation is replaced by

$$
\left(\widehat{D}_{t}^{k+1} p\right)(x, h(x, t), t) \stackrel{(3 \mathrm{D})}{=}-\nabla \cdot A_{1}+\bar{\rho}_{t}^{k+1}
$$

with

$$
A_{1}:=\frac{\partial_{t}^{k+1} \nabla h}{\sqrt{1+|\nabla h|^{2}}}-\frac{\nabla h\left\langle\nabla h, \partial_{t}^{k+1} \nabla h\right\rangle}{\sqrt{1+|\nabla h|^{2}}} .
$$

In the testing procedure $\widehat{D}_{t}^{k+1} p$ is multiplied with

$$
A_{2}:=\partial_{t}^{k+2} h+\delta_{t}^{k+1} \sqrt{1+|\nabla h|^{2}},
$$

and integrated over $S$. We evaluate the two terms that appear additionally in the three-dimensional case. Again, we have to interpret the error terms as divergences. Multiplication of the second term of $A_{1}$ and the first term of $\nabla A_{2}$ yields

$$
-{\sqrt{1+|\nabla h|^{2}}}^{-3} \nabla h\left\langle\nabla h, \partial_{t}^{k+1} \nabla h\right\rangle \cdot \partial_{t}^{k+2} \nabla h=-{\sqrt{1+|\nabla h|^{2}}}^{-3} \partial_{t} \frac{1}{2}\left\langle\nabla h, \partial_{t}^{k+1} \nabla h\right\rangle^{2}+P\left(\bar{\partial}^{k+1} h\right)
$$

for a polynomial $P \in \mathcal{P}^{(1,1)}\left(\bar{\partial}^{k+1} h\right)$. Integration over $[0, T]$ yields the contribution

$$
\left.\int_{S}{\sqrt{1+|\nabla h|^{2}}}^{-3} \frac{1}{2}\left\langle\nabla h, \partial_{t}^{k+1} \nabla h\right\rangle^{2}\right|_{0} ^{T} \leqslant C+\|\nabla h(T)\|_{L^{\infty}(S)}^{2} E^{k+1}(T) .
$$

Since we assumed that $\|\nabla h(T)\|_{L^{\infty}(S)}$ is initially small, it remains small on $[0, T]$, and we can absorb the above error term in the energy estimate.

Concerning the multiplication of the second terms of $A_{1}$ and $\nabla A_{2}$ we evaluate the highest order expression, the contribution $F_{i} \partial_{t}^{k+1} \partial_{x_{i}} h$ of $\delta_{t}^{k+1}$.

$$
-\left\langle\nabla h, \partial_{t}^{k+1} \nabla h\right\rangle\left\langle\nabla h, \nabla\left(F_{i} \partial_{t}^{k+1} \partial_{x_{i}} h\right)\right\rangle=-F_{i} \partial_{x_{i}} \frac{1}{2}\left\langle\nabla h, \partial_{t}^{k+1} \nabla h\right\rangle^{2}+P\left(\bar{\partial}^{k+1} \nabla h, \bar{\partial}^{1} F_{i}\right) .
$$

After an integration by parts this expression is bounded by the energy and the time integral is small.

The product of the first term of $A_{1}$ with the second term of $\nabla A_{2}$ is treated in the same way,

$$
\left\langle\partial_{t}^{k+1} \nabla h, \nabla\left(F_{i} \partial_{t}^{k+1} \partial_{x_{i}} h\right)\right\rangle=F_{i} \partial_{x_{i}} \frac{1}{2}\left|\partial_{t}^{k+1} \nabla h\right|^{2}+P\left(\bar{\partial}^{k+1} \nabla h, \bar{\partial}^{1} F_{i}\right) .
$$


Conclusion of Theorem 1. Based on this analysis of the error terms, the estimate of Theorem 1 follows from (2.18) and (2.20) just as in the two-dimensional case.

\section{Regularity properties of velocity and pressure}

In this section we present the proof of Proposition 3. We will use the results on fractional Sobolev spaces and interpolation that are collected in Appendix.

\subsection{Estimates for the velocity}

Based on the transport equation for $\omega=\operatorname{curl} v$ we will derive estimates for $v$. They will improve the energy estimates by half an order. We will work on a fixed domain using the domain transformation $X(\cdot, t): R \rightarrow \Omega_{t}$. The first step of the proof is an integration lemma: $\operatorname{Given} \operatorname{div}(v)$ and $\operatorname{curl}(v)$, the function $v$ is one order more regular than these data.

Lemma 4. Let the evolution of the domain be given by a function $h$ with bounded norm

$$
h \in L^{\infty}\left(0, T ; H^{k+2}(S)\right) \cap \cdots \cap W^{k+1, \infty}\left(0, T ; H^{1}(S)\right)
$$

and small in the norm

$$
h \in L^{\infty}\left(0, T ; H^{2}(S)\right) \cap L^{\infty}\left(0, T ; C^{0,1}(S)\right) .
$$

We assume furthermore that the vorticity satisfies bounds for

$$
\begin{aligned}
& \bar{D}^{k}(\omega \circ X) \in L^{\infty}\left(0, T ; H^{1 / 2}(R)\right), \\
& \left.\bar{D}^{k}(\omega \circ X)\right|_{S} \in L^{\infty}\left(0, T ; L^{2}(S)\right) .
\end{aligned}
$$

Then the unique solution $v$ of

$$
\begin{array}{ll}
\operatorname{div} v=0, \quad \operatorname{curl} v=\omega & \text { in } \Omega_{t}, \\
v \cdot n=\frac{\partial_{t} h}{\sqrt{1+\left|\nabla_{x} h\right|^{2}}} \quad \text { on } \Gamma_{t},
\end{array}
$$

has bounded derivatives

$$
\begin{aligned}
& \bar{D}^{k} \nabla(v \circ X) \in L^{\infty}\left(0, T ; H^{1 / 2}(R)\right), \\
& \left.\bar{D}^{k} \nabla(v \circ X)\right|_{S} \in L^{\infty}\left(0, T ; L^{2}(S)\right) .
\end{aligned}
$$

Proof. We present the proof in the two-dimensional case. The three-dimensional case follows the same lines and requires only some additional notational effort.

Interior estimates. As a first step we rewrite the equations with an unknown function $u$ living on the fixed domain $R$. We use $B=\left(b_{i j}\right)_{i j}$, the inverse matrix of $D X: R \rightarrow \mathbb{R}^{N \times N}$. On the boundary holds

$$
D X=\left[\begin{array}{cc}
1 & 0 \\
\partial_{x} h & \partial_{y} Y
\end{array}\right], \quad B=\frac{1}{\partial_{y} Y}\left[\begin{array}{cc}
\partial_{y} Y & 0 \\
-\partial_{x} h & 1
\end{array}\right] .
$$

Instead of showing the estimates for $(v \circ X)$, by the regularity of $h$ and $Y$ it is sufficient to show the estimates for the new independent variable $u=\left(u_{i}\right)_{i}$

$$
u_{i}:=\sum_{j} b_{i j} v_{j} \circ X
$$


To verify the equivalence of norms of $v \circ X$ and of $u$ one exploits that for some constant $C$ all functions $f$ and $g$ on $R$ satisfy

$$
\|f \cdot g\|_{H^{1 / 2}(R)} \leqslant C\|f\|_{L^{\infty} \cap H^{3 / 2}}\|g\|_{H^{1 / 2}} .
$$

This follows with an interpolation: the map $K: g \mapsto f \cdot g$ is bounded in $\mathcal{L}\left(L^{2}(R), L^{2}(R)\right)$ and in $\mathcal{L}\left(H^{1}(R), H^{1}(R)\right)$. For the latter we use in three space dimensions the embeddings $H^{1 / 2} \subset L^{3}$ and $H^{1} \subset L^{6}$.

In order to derive equations for $u$ we evaluate

$$
\begin{aligned}
& \sum_{i j} b_{i j} \partial_{i}\left(v_{j} \circ X\right)=\sum_{i j k} b_{i j}\left(\partial_{k} v_{j}\right) \circ X \partial_{i} X_{k}=\sum_{j k}\left(\partial_{k} v_{j}\right) \circ X \delta_{j k}=0, \\
& \begin{aligned}
& \partial_{2} u_{1}-\partial_{1} u_{2}=\partial_{2}\left(\sum_{j} b_{1 j} v_{j} \circ X\right)-\partial_{1}\left(\sum_{j} b_{2 j} v_{j} \circ X\right) \\
&=\sum_{j} \partial_{2} b_{1 j} v_{j} \circ X+\sum_{j k} b_{1 j}\left(\partial_{k} v_{j}\right) \circ X \partial_{2} X_{k}-\sum_{j} \partial_{1} b_{2 j} v_{j} \circ X-\sum_{j k} b_{2 j}\left(\partial_{k} v_{j}\right) \circ X \partial_{1} X_{k}, \\
&\left.u_{2}\right|_{S}=\left.\sum_{j}\left(b_{2 j} v_{j} \circ X\right)\right|_{S}=\frac{1}{\partial_{y} Y}\left(v_{2}-\partial_{x} h v_{1}\right) .
\end{aligned}
\end{aligned}
$$

We find for $u$ the equations

$$
\begin{aligned}
& \operatorname{div} u=\sum_{i j}\left(\partial_{i} b_{i j}\right)(D X \cdot u)_{j} \quad \text { in } R, \\
& \operatorname{curl} u=\omega \circ X+F_{1}\left(\bar{D}_{x} B, \bar{D}_{x}^{2} X\right) \cdot u+F_{2}(B, D X) \cdot \nabla u \text { in } R, \\
& u_{2}=\frac{\partial_{t} h}{\partial_{y} Y} \quad \text { on } S,
\end{aligned}
$$

where $F_{1}, F_{2}$ are polynomials with $F_{2} \equiv 0$ for $h \equiv 0$. We now consider these equations at a fixed time instance $t \in[0, T]$, and omit the index $t$ in the following. We decompose the solution $u$ in two parts, $u=\bar{u}+\tilde{u}$, where $\bar{u}$ solves

$$
\begin{aligned}
& \operatorname{div} \bar{u}=0 \quad \text { in } R, \\
& \operatorname{curl} \bar{u}=0 \quad \text { in } R, \\
& \bar{u} \cdot e_{y}=\frac{\partial_{t} h}{\partial_{y} Y} \quad \text { on } S .
\end{aligned}
$$

We can write $\bar{u}=\nabla \Phi$ for an harmonic function $\Phi$. We infer that $\bar{u}$ has the regularity of continuations of $\partial_{t} h$ and $\partial_{x} h$, i.e.

$$
\|\bar{u}\|_{H^{k+3 / 2}(R)} \leqslant C\left\{\left\|\partial_{t} h\right\|_{H^{k+1}(S)}+\|\nabla h\|_{H^{k+1}(S)}\right\} \leqslant \bar{C} .
$$

The remainder $\tilde{u}$ solves

$$
\begin{aligned}
& \operatorname{div} \tilde{u}=\sum_{i j}\left(\partial_{i} b_{i j}\right)(D X \cdot u)_{j} \quad \text { in } R, \\
& \operatorname{curl} \tilde{u}=\omega \circ X+F_{1}\left(\bar{D}_{x} B, \bar{D}_{x}^{2} X\right) \cdot u+F_{2}(B, D X) \cdot \nabla u \text { in } R, \\
& \tilde{u}_{2}=0 \quad \text { on } S .
\end{aligned}
$$

With the help of standard $L^{2}$ div-curl estimates we conclude that for every $m \leqslant k$

$$
\|\tilde{u}\|_{H^{m+1}(R)} \leqslant C_{1}\|\omega \circ X\|_{H^{m}(R)}+\delta\|u\|_{H^{m+1}(R)}+C_{2}\|u\|_{H^{m}(R)} .
$$


Here $\delta>0$ is arbitrary small for $h$ small in the norm of (3.2). After an interpolation of the standard estimates we conclude additionally

$$
\|\tilde{u}\|_{H^{k+3 / 2}(R)} \leqslant C_{1}\|\omega \circ X\|_{H^{k+1 / 2}(R)}+\delta\|u\|_{H^{k+3 / 2}(R)}+C_{2}\|u\|_{H^{k+1}(R)} .
$$

Here we use again (3.9).

The original equation for $v$ immediately implies an estimate for the $H^{1}$-norm of $v$, which we use to start the induction over $m$ in Eq. (3.11). The induction and Eq. (3.10) yield the desired spatial estimate for $u$.

In order to derive bounds on time derivatives we only have to differentiate the equations for $u$ with respect to time and proceed as above. This concludes the proof of the interior estimates.

Boundary estimates. As a first step we claim that the harmonic function $Y$ satisfies a bound for

$$
\left.\bar{D}^{k+1} \nabla Y\right|_{S} \in L^{\infty}\left(0, T ; L^{2}(S)\right) .
$$

$Y$ is the harmonic extension of the function $h$; this implies bounds for $\bar{D}^{k+1} Y \in L^{\infty}\left(0, T ; H^{3 / 2}(S)\right)$, and therefore a bound for

$$
\left.\bar{D}^{k+1} Y\right|_{S} \in L^{\infty}\left(0, T ; H^{1}(S)\right) .
$$

This implies the result for all $k+2$-nd derivatives that contain a derivative in horizontal direction $x$. For purely normal derivatives we use

$$
\partial_{y}^{k+2} Y=-\partial_{y}^{k} \partial_{x}^{2} Y
$$

and find the result (3.12).

For $u$ we follow a similar path. The trace theorem and the inner estimates yield bounds for

$$
\left.\left(\bar{D}^{k} u\right)\right|_{S} \in L^{\infty}\left(0, T ; H^{1}(S)\right),
$$

which is a bound for $\left.\partial_{x} \bar{D}^{k} u\right|_{S} \in L^{\infty}\left(0, T ; L^{2}(S)\right)$. For normal derivatives of $\bar{D}^{k} u$ we have to exploit the equations for divergence and curl.

$$
\partial_{y} \bar{D}^{k} u_{2}=\bar{D}^{k} \partial_{y} u_{2}=-\bar{D}^{k} \partial_{x} u_{1}+\bar{D}^{k} \operatorname{div} u \in L^{\infty}\left(0, T ; L^{2}(S)\right) .
$$

We used (3.12) here. For derivatives of $u_{1}$ on the boundary we calculate

$$
\partial_{y} \bar{D}^{k} u_{1}=\bar{D}^{k} \partial_{y} u_{1}=\bar{D}^{k} \partial_{x} u_{2}+\bar{D}^{k} \operatorname{curl} u .
$$

Using (3.4), (3.12), and an iterative improvement as in (3.11) this concludes the proof.

In order to describe our compatibility assumption on the initial values we have to introduce the concept of formal time derivatives. Given only the initial values $\left(v^{0}, h^{0}\right)$ we can calculate the initial pressure $p^{0}$ from its boundary values (determined by $h^{0}$ and (1.4)), and $\Delta p^{0}=\operatorname{div}\left(\left(v^{0} \cdot \nabla\right) v^{0}\right)$. The initial pressure now determines the initial time derivative of $v$ by (1.1). The boundary values of $v^{0}$ determine the initial time derivative of $h$ by (1.3). Differentiating the equations we can also find higher time derivatives; they depend only on the initial values $\left(v^{0}, h^{0}\right)$ and are denoted by $\tilde{\partial}_{t}^{m} v^{0}, \tilde{\partial}_{t}^{m} p^{0}$, and $\tilde{\partial}_{t}^{m} h^{0}$. From Eq. (1.7) we can determine $\tilde{\partial}_{t}^{m} \omega^{0}$.

Assumption 5. Let the initial values be such that the formal time derivatives for $v, p, h$ and $\omega$ are bounded in

$$
\begin{array}{ll}
\tilde{\partial}_{t}^{m} v^{0} \in H^{k+3 / 2-m}\left(\Omega_{0}\right) & \forall m \leqslant k, \quad \tilde{\partial}_{t}^{k+1} v^{0} \in L^{2}\left(\Omega_{0}\right), \\
\left.\left(\tilde{\partial}_{t}^{m} \nabla v^{0}\right) \circ X\right|_{S} \in H^{k-m}(S) & \forall m \leqslant k, \\
\tilde{\partial}_{t}^{m} h^{0} \in H^{k+2-m}(S) & \forall m \leqslant k+1 .
\end{array}
$$


Note that the above estimates automatically implies bounds for $\omega=\operatorname{curl} v$ :

$$
\begin{array}{ll}
\tilde{\partial}_{t}^{m} \omega^{0} \in H^{k+1 / 2-m}\left(\Omega_{0}\right) & \forall m \leqslant k, \\
\left.\left(\tilde{\partial}_{t}^{m} \omega^{0}\right) \circ X\right|_{S} \in H^{k-m}(S) & \forall m \leqslant k .
\end{array}
$$

The next step in our analysis is to exploit the vorticity equation.

Lemma 6. We consider a time dependent domain, given by a function $h$ with bounded norm

$$
h \in L^{\infty}\left(0, T ; H^{k+2}(S)\right) \cap \cdots \cap W^{k+1, \infty}\left(0, T ; H^{1}(S)\right) .
$$

Let $\omega=$ curl $v$ solve (1.7) and assume that the initial values are bounded in the norms of Assumption 5. for

Then, for a possibly smaller $T>0$, the vorticity $\omega \circ X$ satisfies bounds in the spaces of (3.3), (3.4), i.e. bounds

$$
\begin{aligned}
& \bar{D}^{k}(\omega \circ X) \in L^{\infty}\left(0, T ; H^{1 / 2}(R)\right), \\
& \left.\bar{D}^{k}(\omega \circ X)\right|_{S} \in L^{\infty}\left(0, T ; L^{2}(S)\right) .
\end{aligned}
$$

Proof. The primary goal is to find a priori estimates for the transport equation

$$
\begin{aligned}
& \partial_{t} \omega+(v \cdot \nabla) \omega=f \quad \text { in } \Omega_{t}, \\
& \omega(0)=\omega_{0} \quad \text { in } \Omega_{0},
\end{aligned}
$$

for a given domain evolution and for a given velocity field $v$ satisfying the boundary condition (1.3).

Interior estimates. We differentiate the transport equation $m$ times with respect to $t$ and obtain

$$
\left[\partial_{t}+(v \cdot \nabla)\right] \widehat{D}_{t}^{m} \omega=\left[\partial_{t}, \widehat{D}_{t}^{m}\right] \omega+\left[(v \cdot \nabla), \widehat{D}_{t}^{m}\right] \omega+\widehat{D}_{t}^{m} f=\widehat{D}_{t}^{m} f+F\left(\bar{D}^{m} Y_{t}, \bar{D}^{m} v, \bar{D}^{m} \omega\right),
$$

where $F$ is a polynomial that is linear in the set of highest and second but highest derivatives. Multiplication of Eq. (3.14) with $\widehat{D}_{t}^{m} \omega$ and integration over $\Omega_{t}$ yields with the transport theorem

$$
\left.\int_{\Omega_{t}} \frac{1}{2}\left|\widehat{D}_{t}^{m} \omega\right|^{2}\right|_{t=0} ^{\tau} \leqslant C \sup _{t \in(0, T)}\left\{\int_{\Omega_{t}}\left|\widehat{D}_{t}^{m} \omega\right|^{2}(\cdot, t)\right\}^{1 / 2}\left\|\widehat{D}_{t}^{m} f \circ X+F\left(\bar{D}^{m} Y_{t}, \bar{D}^{m} v, \bar{D}^{m} \omega\right) \circ X\right\|_{L^{1}\left(0, T ; L^{2}\right)} .
$$

The same calculation can be done with spatial derivatives of $\omega$. We will use the estimate with $m=k$ and $m=$ $k+1$, in the latter case we assume that at least one derivative is spatial. Using the equivalence of the two norms $\left\|\bar{D}^{m} \Theta \circ X\right\|_{L^{2}}$ and $\left\|\bar{D}^{m}(\Theta \circ X)\right\|_{L^{2}}$ for $\Theta$, we find by the regularity of $h$

$$
\begin{aligned}
\left\|\bar{D}^{m}(\omega \circ X)\right\|_{L^{\infty} L^{2}} \leqslant & C_{v}\left\{\left\|\bar{D}^{m} \omega(0)\right\|_{L^{2}}+\left\|\bar{D}^{m}(f \circ X)\right\|_{L^{1} L^{2}}\right. \\
& \left.+1+\left\|\bar{D}^{m}(v \circ X)\right\|_{L^{1} L^{2}}+\left\|\bar{D}^{m}(\omega \circ X)\right\|_{L^{1} L^{2}}\right\} .
\end{aligned}
$$

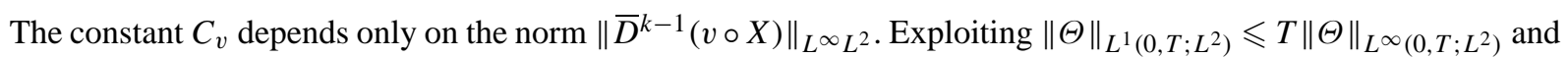
choosing $T$ small (depending on $C_{v}$ ) we can absorb the last term into the left-hand side.

$$
\left\|\bar{D}^{m}(\omega \circ X)\right\|_{L^{\infty} L^{2}} \leqslant C C_{v}\left\{\left\|\bar{D}^{m} \omega(0)\right\|_{L^{2}}+\left\|\bar{D}^{m}(f \circ X)\right\|_{L^{1} L^{2}}+1+\left\|\bar{D}^{m}(v \circ X)\right\|_{L^{1} L^{2}}\right\} .
$$

We next interpolate this estimate for $m=k$ and $m=k+1$ in order to obtain an estimate of $k$-th derivatives in $H^{1 / 2}$.

(a) Dependence on $\omega_{0}$. We study the case $f \equiv 0$ and $v$ fixed in order to study the (linear) dependence of the solution $\omega$ upon $\omega_{0}$. The map

$$
J: \omega_{0} \circ X_{0} \mapsto\left((\omega \circ X)(0), \ldots, \tilde{\partial}_{t}^{k}(\omega \circ X)(0)\right)
$$


is bounded in the function spaces

$$
J: H^{m}(R) \rightarrow H^{m}(R) \times \cdots \times H^{m-k}(R)
$$

for $m=k$ and $m=k+1$. (3.16) yields that for every $\tau \leqslant T$ and $l \leqslant k$ the map

$$
S_{l}: H^{m}(R) \times \cdots \times H^{m-k}(R) \ni\left((\omega \circ X)(0), \ldots, \tilde{\partial}_{t}^{k}(\omega \circ X)(0)\right) \mapsto \partial_{t}^{l}(\omega \circ X)(\tau) \in H^{m-l}(R)
$$

is bounded. The interpolation between $k$ and $k+1$ yields that the linear maps $S_{l} \circ J$ are also bounded as maps

$$
S_{l} \circ J: H^{k+1 / 2}(R) \rightarrow H^{k+1 / 2-l}(R) .
$$

This provides the regularity in dependence of the initial data.

(b) Dependence on $f$. We now assume $\omega_{0}=0$ and investigate the dependence of $\omega$ on $f$. For fixed $f$ with bounded norms

$$
\bar{D}^{k}(f \circ X) \in L^{1}\left(0, T ; H^{m-k}(R)\right),
$$

we automatically have a bound for

$$
\partial_{t}^{l} f(0) \circ X_{0} \in H^{m-1-l}(R)
$$

for every $l \leqslant k-1$. These expressions enter in the calculation of formal time derivatives of $\omega$ in $t=0$. The estimate (3.16) yields that for every $l \leqslant k$ the linear map

$$
U_{l}:\left(f \circ X, \ldots, \partial_{t}^{k}(f \circ X)\right) \mapsto \partial_{t}^{l}(\omega \circ X)(\tau)
$$

is bounded in the spaces

$$
U_{l}: L^{1} H^{m}(R) \times \cdots \times L^{1} H^{m-k}(R) \rightarrow H^{m-l}(R) .
$$

With another interpolation between $m=k$ and $m=k+1$ we find the result for the $f$-dependence.

Our interpolated estimate now reads

$$
\begin{aligned}
& \left\|\bar{D}^{k}(\omega \circ X)\right\|_{L^{\infty} H^{1 / 2}(R)} \\
& \quad \leqslant C C_{v}\left\{\left\|\bar{D}^{k} \omega(0) \circ X_{0}\right\|_{H^{1 / 2}(R)}+\left\|\bar{D}^{k}(f \circ X)\right\|_{L^{1} H^{1 / 2}(R)}+\left\|\bar{D}^{k} \nabla(v \circ X)\right\|_{L^{1} L^{2}(R)}\right\} .
\end{aligned}
$$

In order to treat the norm of $v$ on the right-hand side we use Lemma 4.

$$
\left\|\bar{D}^{k} \nabla(v \circ X)\right\|_{L^{1} L^{2}} \leqslant T\left\|\bar{D}^{k} \nabla(v \circ X)\right\|_{L^{\infty} L^{2}} \leqslant T C\left(1+\left\|\bar{D}^{k}(\omega \circ X)\right\|_{L^{\infty} H^{1 / 2}}+\left\|\left.\bar{D}^{k}(\omega \circ X)\right|_{S}\right\|_{L^{\infty} L^{2}}\right) .
$$

For small $T$ and with the boundary estimate below we can absorb the last term in estimate (3.18).

In the two-dimensional case $f$ vanishes; in the three-dimensional case we have $f=(\omega \cdot \nabla) v$ and we must exploit Lemma 4 in its full strength. It yields

$$
\begin{aligned}
\left\|\bar{D}^{k}(f \circ X)\right\|_{L^{1} H^{1 / 2}} & \leqslant C_{v}^{\prime}\left\|\bar{D}^{k} \nabla(v \circ X)\right\|_{L^{1} H^{1 / 2}} \\
& \leqslant T C_{v}^{\prime}\left(1+\left\|\bar{D}^{k}(\omega \circ X)\right\|_{L^{\infty} H^{1 / 2}}+\left\|\left.\bar{D}^{k}(\omega \circ X)\right|_{S}\right\|_{L^{\infty} L^{2}(S)}\right) .
\end{aligned}
$$

Together with the boundary estimate below, (3.18) provides an estimate for

$$
\zeta:=\left\|\bar{D}^{k}(\omega \circ X)\right\|_{L^{\infty} H^{1 / 2}(R)}+\left\|\left.\bar{D}^{k}(\omega \circ X)\right|_{S}\right\|_{L^{\infty} L^{2}(S)}
$$

of the form

$$
\zeta \leqslant C_{v}\left(C_{1}+C_{2} T \zeta\right)
$$


We exploit that $C_{v}$ contains only lower order derivatives of $v$ and can therefore be estimated in terms of its initial values and an integral over its temporal derivative, whence

$$
C_{v} \leqslant C_{3}+C_{4} T \Phi(\zeta)
$$

for some polynomial $\Phi$. We combine the last two inequalities and find with a new constant $C_{1}$ and a new polynomial $\Phi$

$$
\zeta \leqslant C_{1}+T \Phi(\zeta)
$$

Enlarging $C_{1}$ if necessary, we can assume that on a small time interval $(0, \varepsilon)$ there holds $\zeta \leqslant C_{1}$ (we assumed that the initial values are smooth). Choosing $T$ small (depending only on the norms of the initial values as in Assumption 5), we conclude that $\zeta$ can never exceed the value $C_{1}+1$.

Boundary estimates. As for the interior estimates we start from (3.14) and multiply again with $\widehat{D}_{t}^{k} \omega$, but this time we will integrate only over the boundaries $\Gamma_{t}$. We have to choose an appropriate parametrization of $\Gamma_{t}$, namely

$$
\gamma_{t}: S \rightarrow \mathbb{R}^{N} \text { with } \partial_{t} \gamma_{t}(x)=v\left(\gamma_{t}(x), t\right) .
$$

With this parametrization we find

$$
\frac{\mathrm{d}}{\mathrm{d} t} \int_{S} g(\cdot, t) \circ \gamma_{t}=\int_{S}\left[\frac{D}{D t} g(\cdot, t)\right] \circ \gamma_{t} .
$$

We set $g=\frac{1}{2}\left|\widehat{D}_{t}^{k} \omega\right|^{2}$ and integrate over time.

$$
\begin{aligned}
& \left.\int_{S} \frac{1}{2}\left|\widehat{D}_{t}^{k} \omega\right|^{2} \circ \gamma_{t}\right|_{t=0} ^{\tau} \\
& \quad=\int_{0}^{\tau} \int_{S}\left[\widehat{D}_{t}^{k} \omega \cdot\left(\widehat{D}_{t}^{k} f+F\left(\bar{D}^{k} Y_{t}, \bar{D}^{k} v, \bar{D}^{k} \omega\right)\right)\right] \circ \gamma \\
& \quad \leqslant C \sup _{t \in(0, T)}\left\{\int_{S}\left|\widehat{D}_{t}^{k} \omega\right|^{2} \circ X\right\}^{1 / 2}\left\|\left.\left(\widehat{D}_{t}^{k} f \circ X+F\left(\bar{D}^{k} Y_{t}, \bar{D}^{k} v, \bar{D}^{k} \omega\right) \circ X\right)\right|_{S}\right\|_{L^{1}\left(0, T ; L^{2}(S)\right)}
\end{aligned}
$$

by equivalence of $L^{2}$-norms with the different parametrizations. The same calculation can be done for spatial derivatives. Again using norm equivalence we find

$$
\begin{aligned}
& \left\|\bar{D}^{k}(\omega \circ X)\right\|_{L^{\infty}\left(0, T ; L^{2}(S)\right)} \\
& \quad \leqslant C_{v}\left\{\left\|\bar{D}^{k} \omega(0) \circ X\right\|_{L^{2}(S)}+\left\|\bar{D}^{k}(f \circ X)\right\|_{L^{1} L^{2}(S)}+\left\|\bar{D}^{k}(v \circ X)\right\|_{L^{1} L^{2}(S)}+\left\|\bar{D}^{k}(\omega \circ X)\right\|_{L^{1} L^{2}(S)}\right\} .
\end{aligned}
$$

The proof is finished as in the lines after (3.18): the last term can be absorbed in the left-hand side, the second but last term is of lower order, for the term containing $f$ we exploit the boundary estimates of Lemma 4.

\subsection{Estimates for the pressure}

The estimates for the pressure are based not only on the regularity of $v$ expressed in (3.5), but also on the energy estimates

$$
\left\|\partial_{t}^{k+1}(v \circ X)\right\|_{L^{\infty}\left(0, T ; L^{2}(R)\right)}+\left\|\partial_{t}^{k+1} \partial_{x} h\right\|_{L^{\infty}\left(0, T ; L^{2}(S)\right)} \leqslant C .
$$


We write the Euler equation as $\nabla p=-\partial_{t} v-(v \nabla) v$. The regularity of $v$ implies that the right-hand side has the $k$-th time derivative bounded in $L^{\infty}\left(0, T ; L^{2}(R)\right)$, and mixed (or purely spatial) $k$-th derivatives bounded in $L^{\infty}\left(0, T ; H^{1 / 2}(R)\right)$. We infer the following estimate for the pressure.

$$
\|p \circ X\|_{L^{\infty}\left(0, T ; H^{k+3 / 2}(R)\right)}+\cdots+\|p \circ X\|_{W^{k-1, \infty}\left(0, T ; H^{2+1 / 2}(R)\right)}+\|p \circ X\|_{W^{k, \infty}\left(0, T ; H^{1}(R)\right)} \leqslant C_{0} .
$$

It remains to show the estimate for the $k+1$ time derivative which is verified in the following lemma.

Lemma 7. Under the assumptions of Proposition 3 the pressure satisfies the estimate

$$
\|p \circ X\|_{W^{k+1, \infty}\left(0, T ; H_{*}^{-1 / 2}(R)\right)} \leqslant C,
$$

where $C$ depends only on the norm of $h$ and on the constant $C_{0}$ of (3.20). In this estimate $H_{*}^{-1 / 2}(R)$ denotes the space of functions $g$ of the form

$$
g=Q_{0}+\sum_{j=1}^{N-1} \partial_{x_{j}} Q_{j},
$$

with $Q_{0} \in L^{2}(R), Q_{1}, \ldots, Q_{N-1} \in H^{1 / 2}(R)$.

Proof. Step 1. An equation for $u:=\partial_{t}^{k+1}(p \circ X)$. The first step in the proof is to derive an equation for $u$. We begin by transforming the Laplace operator on the domain $\Omega_{t}$ to the domain $R$, that is, for functions of the form $f \circ X: R \rightarrow \mathbb{R}$. We set $B=\left(b_{i j}\right)_{i j}=(D X)^{-1}$ and $A=B B^{\mathrm{T}}$. Then for $f \in C^{2}(\Omega, \mathbb{R})$ and $X: R \rightarrow \Omega$ one-to-one we can calculate

$$
\begin{aligned}
\nabla \cdot(A \nabla(f \circ X)) & =\sum_{i j k l} \partial_{i}\left(b_{i l} b_{j l}\left(\partial_{k} f\right) \circ X \partial_{j} X_{k}\right)=\sum_{i k} \partial_{i}\left(b_{i k}\left(\partial_{k} f\right) \circ X\right) \\
& =\sum_{i k l} b_{i k}\left(\partial_{l} \partial_{k} f\right) \circ X \partial_{i} X_{l}+\sum_{i k} \partial_{i} b_{i k}\left(\partial_{k} f\right) \circ X \\
& =\sum_{k}\left(\partial_{k}^{2} f\right) \circ X+\sum_{i k l} \partial_{i} b_{i k} \partial_{l}(f \circ X) b_{l k} \\
& =(\Delta f) \circ X+\operatorname{div} B \cdot B^{\mathrm{T}} \cdot \nabla(f \circ X) .
\end{aligned}
$$

With the operator $\mathcal{L} w:=\nabla \cdot(A \nabla w)-\operatorname{div} B \cdot B^{\mathrm{T}} \cdot \nabla w$ we can write this equality as

$$
\mathcal{L}(f \circ X)=(\Delta f) \circ X .
$$

We can now derive the equation for $u$, exploiting $\Delta p=-\operatorname{div}[(v \cdot \nabla) v]=-\sum_{i j} \partial_{i} v_{j} \partial_{j} v_{i}$.

$$
\begin{aligned}
\mathcal{L} u= & \mathcal{L} \partial_{t}^{k+1}(p \circ X) \\
= & \mathcal{L}\left(\partial_{t}^{k+1} p \circ X\right)+\mathcal{L}\left(\sum_{i} k \partial_{t}^{k} \partial_{i} p \circ X \partial_{t} X_{i}\right)+\cdots+\mathcal{L}\left(\sum_{i} \partial_{i} p \circ X \partial_{t}^{k+1} X_{i}\right) \\
= & \left(\Delta \partial_{t}^{k+1} p\right) \circ X+k\left(\Delta \sum_{i} \partial_{t}^{k} \partial_{i} p \partial_{t} X_{i} \circ X^{-1}\right) \circ X+\cdots+\mathcal{L}\left(\sum_{i} \partial_{i} p \circ X \partial_{t}^{k+1} X_{i}\right) \\
= & -\left(\partial_{t}^{k+1} \sum_{i j} \partial_{i} v_{j} \partial_{j} v_{i}\right) \circ X-k \sum_{i}\left(\partial_{t}^{k} \partial_{i}\left[\sum_{l j} \partial_{l} v_{j} \partial_{j} v_{l}\right] \partial_{t} X_{i} \circ X^{-1}\right) \circ X+\cdots \\
& +\mathcal{L}\left(\sum_{i} \partial_{i} p \circ X \partial_{t}^{k+1} X_{i}\right) .
\end{aligned}
$$


We conclude that

$$
\mathcal{L} u=\operatorname{div} F_{1}+F_{2},
$$

with $F_{2}$ in $L^{\infty}\left(0, T ; L^{2}(R)\right)$. The function $F_{1}$ includes the term $\partial_{t}^{k+1} v$, first derivatives of $\partial_{t}^{k} v$, first derivatives of $\partial_{t}^{k+1} X_{i}$, and first derivatives of $\partial_{t}^{k} p$. Therefore $F_{1} \in L^{\infty}\left(0, T ; L^{2}(R)\right)$.

The boundary values of $u$ are

$$
u=\partial_{t}^{k+1} \nabla \cdot\left(\frac{\nabla h}{\sqrt{1+|\nabla h|^{2}}}\right) .
$$

Step 2. A decomposition of $u$. In this and the next step of the proof we do not indicate the boundary conditions on the lower boundary of the rectangle $R$.

We decompose $u$ into a boundary contribution and a remainder of higher regularity,

$$
u=\bar{u}+\tilde{u},
$$

with $\bar{u}: R \times I \rightarrow \mathbb{R}$ being the solution of

$$
\begin{aligned}
& \mathcal{L} \bar{u}=0 \quad \text { in } R, \\
& \bar{u}=\nabla \cdot \partial_{t}^{k+1}\left(\frac{\nabla h}{\sqrt{1+|\nabla h|^{2}}}\right)=: \nabla \cdot g \quad \text { on } S .
\end{aligned}
$$

Note that we have a bound for $g \in L^{\infty}\left(0, T ; L^{2}(S)\right)$. The remainder $\tilde{u}$ satisfies by linearity of the equations

$$
\begin{aligned}
& \mathcal{L} \tilde{u}=\operatorname{div} F_{1}+F_{2} \quad \text { in } R, \\
& \tilde{u}=0 \quad \text { on } S .
\end{aligned}
$$

Testing this equation with $\tilde{u}$ we immediately conclude the regularity $\tilde{u} \in L^{\infty}\left(0, T ; H^{1}(R)\right)$. It remains to verify a bound for

$$
\bar{u} \in L^{\infty}\left(0, T ; H_{*}^{-1 / 2}(R)\right) .
$$

We try to approximate $\bar{u}$ as $\sum_{i=1}^{N-1} \partial_{i} U_{i}$ with $U_{i}$ solving

$$
\begin{array}{ll}
\mathcal{L} U_{i}=0 & \text { in } R, \\
U_{i}=g_{i} & \text { on } S .
\end{array}
$$

$U_{i}$ has the regularity $U_{i} \in L^{\infty}\left(0, T ; H^{1 / 2}(R)\right)$, therefore the contribution $\sum_{i=1}^{N-1} \partial_{i} U_{i}$ has the desired estimate in $L^{\infty}\left(0, T ; H_{*}^{-1 / 2}(R)\right)$. The remainder $V:=\bar{u}-\sum_{i=1}^{N-1} \partial_{i} U_{i}$ solves the system

$$
\begin{aligned}
& \mathcal{L} V=\left(\sum_{i=1}^{N-1} \partial_{i} \mathcal{L}\right) U_{i} \quad \text { in } R, \\
& V=0 \quad \text { on } S .
\end{aligned}
$$

It remains to show that $V \in L^{\infty}\left(0, T ; L^{2}(R)\right)$ is bounded.

Step 3. Regularity of $V$. We have to study solutions $V$ of

$$
\begin{aligned}
& \nabla \cdot(A \nabla V)=\nabla \cdot(B \nabla U) \text { in } R, \\
& V=0 \quad \text { on } S,
\end{aligned}
$$

with smooth $A$ and $B, U \in H^{1 / 2}(R)$, and $\left.U\right|_{S}=g \in L^{2}(S)$. In order to show a bound for $V \in L^{2}(R)$ we take an arbitrary $w \in L^{2}(R)$ and study the $L^{2}$-product $\langle V, w\rangle$.

Given $w$ we solve the dual problem

$$
\begin{aligned}
& \partial_{j}\left(a_{i j} \partial_{i} \varphi\right)=w \text { in } R, \\
& \varphi=0 \text { on } S .
\end{aligned}
$$


Then with $\psi=\left(a_{i j} \partial_{i} \varphi\right)_{j}$ we find

$$
\begin{aligned}
\int_{R} V w & =\int_{R} V \operatorname{div}(\psi)=-\int_{R} \nabla V \cdot \psi=\int_{R} \partial_{i}\left(a_{i j} \partial_{j} V\right) \varphi=-\int_{R}(B \nabla U) \cdot \nabla \varphi \\
& =\int_{R} U \nabla \cdot\left(B^{\mathrm{T}} \nabla \varphi\right)+\int_{S} U n \cdot\left(B^{\mathrm{T}} \nabla \varphi\right) \leqslant C\left(\|U\|_{L^{2}(R)}+\left\|\left.U\right|_{S}\right\|_{L^{2}(S)}\right)\|\varphi\|_{H^{2}(R)} .
\end{aligned}
$$

Since $\|\varphi\|_{H^{2}(R)}$ can be bounded in terms of $\|w\|_{L^{2}(R)}$, this yields an $L^{2}$ estimate for $V$,

$$
\|V\|_{L^{2}(R)} \leqslant C\left(\|U\|_{L^{2}(R)}+\left\|\left.U\right|_{S}\right\|_{L^{2}(S)}\right) .
$$

This concludes the proof.

\section{A Navier-Stokes approximation}

In the previous sections we have derived a priori estimates for smooth solutions of the incompressible Euler equations $(E)$. Once we can approximate $(E)$ by a system $\left(E_{\varepsilon}\right)$ that has smooth solutions, we can conclude the existence of solutions to $(E)$ - we only have to verify that solutions of $\left(E_{\varepsilon}\right)$ satisfy the same a priori estimates.

Our estimates are derived using both, the energy estimates for $v$ and $h$, and the estimates for the vorticity $\omega$. If we approximate the equations for $v$ in a generic way, e.g. with a Galerkin scheme, we lose the structure in the equations for $\omega$. Then the a priori estimates can not be reproduced for the approximation. Our solution to this problem is to use an approximating system $\left(E_{\varepsilon}\right)$ that has the same structure as $(E)$ - we choose the Navier-Stokes equations.

In this section we do all calculations in the two-dimensional case $N=2$. The method works in the same way for $N=3$.

The Navier-Stokes equations read

$$
\begin{aligned}
& \partial_{t} v+(v \cdot \nabla) v+\nabla p=\varepsilon \Delta v, \\
& \operatorname{div} v=0,
\end{aligned}
$$

in the time dependent domain $\Omega_{t}$. Two of the physical boundary conditions are the kinematic relation and balance of normal forces.

$$
\begin{aligned}
& \partial_{t} h-v_{2}+\partial_{x} h \cdot v_{1}=0, \\
& \partial_{x} \cdot\left(\frac{\partial_{x} h}{\sqrt{1+\left|\partial_{x} h\right|^{2}}}\right)+p=2 \varepsilon\left(\partial_{n} v\right) \cdot n .
\end{aligned}
$$

On the fixed boundary we use the normal condition $v \cdot n=0$. It remains to choose tangential boundary conditions on upper and lower boundary. We will not use the physical equation, but introduce an artificial condition: we impose on the free boundary and on the bottom the condition

$$
\omega=0 .
$$

The equation for the vorticity $\omega:=\operatorname{curl} v$ reads

$$
\partial_{t} \omega+(v \cdot \nabla) \omega=\varepsilon \Delta \omega .
$$

The boundary condition (4.5) helps to exploit this vorticity equation. Note that with a no-slip condition on the bottom we could not hope to approximate the solution of the Euler equations in smooth function spaces. 
We will derive estimates for the Navier-Stokes system by following the ideas used for the Euler equations. We will see that Proposition 3 remains valid: The reconstruction of $v$ from $\omega$ in Lemma 4 uses only the incompressibility and the kinematic condition. The regularity properties of $\omega$ in Lemma 6 can be reproduced due to our boundary condition. Lemma 7 remains valid with slight modifications in the statement and in the proof.

The a priori estimates follow if we can repeat the differentiated energy estimates of Section 2 for solutions of the Navier-Stokes equations. This is not obvious due to the artificial tangential boundary condition.

\subsection{First energy estimate}

Multiplication of (4.1) with $v$ and integrating over $\Omega_{t}$ yields

$$
\int_{\Omega_{t}}\left(\partial_{t}+v \cdot \nabla\right) \frac{1}{2}|v|^{2}+\int_{\Gamma_{t}} p v_{n}=-\varepsilon \int_{\Omega_{t}}|\nabla v|^{2}+\varepsilon \int_{\Gamma_{t}} \partial_{n} v \cdot v .
$$

By the transport theorem and by expanding the product under the last integral we find with the tangential unit vector $\tau$

$$
\frac{\mathrm{d}}{\mathrm{d} t} \frac{1}{2} \int_{\Omega_{t}}|v|^{2}+\varepsilon \int_{\Omega_{t}}|\nabla v|^{2}+\int_{\Gamma_{t}}\left[p-\varepsilon\left(\partial_{n} v\right)_{n}\right] v_{n}=\varepsilon \int_{\Gamma_{t}}\left(\partial_{n} v\right)_{\tau} v_{\tau} .
$$

We calculate for the right-hand side

$$
\begin{aligned}
\int_{\Gamma_{t}}\left(\partial_{n} v\right)_{\tau} v_{\tau} \stackrel{\omega=0}{=} \int_{\Gamma_{t}}\left(\partial_{\tau} v\right)_{n} v_{\tau}=\int_{\Gamma_{t}}\left[\partial_{\tau}\left(v_{n}\right)-v \cdot \partial_{\tau} n\right] v_{\tau} \\
\stackrel{(\text { P.I. })}{=}-\int_{\Gamma_{t}} v_{n} \partial_{\tau}\left(v_{\tau}\right)-\int_{\Gamma_{t}} v \cdot\left(\partial_{\tau} n\right) v_{\tau} \\
=\int_{\Gamma_{t}} v_{n}\left(\partial_{n} v\right)_{n}-\int_{\Gamma_{t}} v_{n} \partial_{\tau} \tau \cdot v-\int_{\Gamma_{t}} v \cdot\left(\partial_{\tau} n\right) v_{\tau} .
\end{aligned}
$$

The energy estimate is now

$$
\partial_{t} \frac{1}{2} \int_{\Omega_{t}}|v|^{2}+\partial_{t} \mathcal{H}^{N-1}\left(\Gamma_{t}\right)+\varepsilon \int_{\Omega_{t}}|\nabla v|^{2}=-\int_{\Gamma_{t}} v_{n} \partial_{\tau} \tau \cdot v-\int_{\Gamma_{t}} v \cdot\left(\partial_{\tau} n\right) v_{\tau} .
$$

This equation does not yield estimates for the energy since the right-hand side contains second derivatives of $h$. Nevertheless, for derivatives of the solution we can expect that the error terms on the right-hand side are of lower order.

\subsection{Higher order energy estimates}

Just as we did for the Euler equations we can differentiate the Navier-Stokes equations with respect to time. Again, in order to find total time derivatives of boundary values we use the vertical material derivative $\widehat{D}_{t}^{1}=$ $\partial_{t}+Y_{t} \partial_{y}$.

The equation for $v_{t}=\widehat{D}_{t}^{1} v$ and $p_{t}=\widehat{D}_{t}^{1} p$ reads

$$
\frac{D}{D t} v_{t}=-\nabla p_{t}+\varepsilon \Delta v_{t}+f_{t}^{1}+\varepsilon F_{t}^{1}
$$

It coincides with the Euler case except for the expression $F_{t}^{1}$, produced by the commutator

$$
F_{t}^{1}=-\left[\Delta, Y_{t} \partial_{y}\right] v
$$


The divergence $\operatorname{div} v_{t}=g_{t}^{1}$ remains unchanged. The energy estimate reads in its first form

$$
\partial_{t} \frac{1}{2} \int_{\Omega_{t}}\left|v_{t}\right|^{2}+\int_{\Gamma} p_{t} v_{t} \cdot n+\varepsilon \int_{\Omega_{t}}\left|\nabla v_{t}\right|^{2}-\varepsilon \int_{\Gamma} \partial_{n} v_{t} \cdot v_{t}=\int_{\Omega_{t}}\left(f_{t}^{1}+\varepsilon F_{t}^{1}\right) \cdot v_{t}+\int_{\Omega_{t}} g_{t}^{1} p_{t} .
$$

In order to recover the expression $p_{t}-2 \varepsilon\left(\partial_{n} v_{t}\right)_{n}$ as a factor of $\left(v_{t}\right)_{n}$ we have to follow the ideas of Subsection 4.1. Differentiating the equation $\omega=0$ yields

$$
\widehat{D}_{t}\left[\left(\partial_{n} v\right)_{\tau}\right]=\widehat{D}_{t}\left[\left(\partial_{\tau} v\right)_{n}\right]
$$

or, using the Leibniz rule

$$
\left(\widehat{D}_{t} n \cdot \nabla v\right)_{\tau}+\left(\partial_{n} v_{t}\right)_{\tau}-\left(\partial_{n} Y_{t} \partial_{y} v\right)_{\tau}+\left(\partial_{n} v\right) \cdot \widehat{D}_{t} \tau=\left(\widehat{D}_{t} \tau \cdot \nabla v\right)_{n}+\left(\partial_{\tau} v_{t}\right)_{n}-\left(\partial_{\tau} Y_{t} \partial_{y} v\right)_{n}+\left(\partial_{\tau} v\right) \cdot \widehat{D}_{t} n .
$$

We can therefore replace one integral by

$$
\int_{\Gamma}\left(\partial_{n} v_{t}\right)_{\tau}\left(v_{t}\right)_{\tau}=\int_{\Gamma}\left(\partial_{\tau} v_{t}\right)_{n}\left(v_{t}\right)_{\tau}+\int_{\Gamma} \bar{\pi}_{t}^{1}
$$

with the error term

$$
\bar{\pi}_{t}^{1}=\left(v_{t}\right)_{\tau}\left[\left(\widehat{D}_{t} \tau \cdot \nabla v\right)_{n}-\left(\partial_{\tau} Y_{t} \partial_{y} v\right)_{n}+\left(\partial_{\tau} v\right) \cdot \widehat{D}_{t} n-\left(\widehat{D}_{t} n \cdot \nabla v\right)_{\tau}+\left(\partial_{n} Y_{t} \partial_{y} v\right)_{\tau}-\left(\partial_{n} v\right) \cdot \widehat{D}_{t} \tau\right] .
$$

We proceed with an integration by parts of the operator $\partial_{\tau}$.

$$
\int_{\Gamma}\left(\partial_{\tau} v_{t}\right)_{n}\left(v_{t}\right)_{\tau}=-\int_{\Gamma}\left(v_{t}\right)_{n}\left(\partial_{\tau} v_{t}\right)_{\tau}+\int_{\Gamma} \tilde{\pi}_{t}^{1},
$$

with the error term

$$
\tilde{\pi}_{t}^{1}=-\left(v_{t}\right)_{n} \partial_{\tau} \tau \cdot v_{t}-v_{t} \cdot \partial_{\tau} n\left(v_{t}\right)_{\tau} .
$$

The third step was to exploit incompressibility, $\left(\partial_{\tau} v\right)_{\tau}=-\left(\partial_{n} v\right)_{n}$. An application of $\widehat{D}_{t}$ yields

$$
\left(\widehat{D}_{t} \tau \cdot \nabla v\right)_{\tau}+\left(\partial_{\tau} v_{t}\right)_{\tau}-\left(\partial_{\tau} Y_{t} \partial_{y} v\right)_{\tau}+\left(\partial_{\tau} v\right) \cdot \widehat{D}_{t} \tau=-\left(\widehat{D}_{t} n \cdot \nabla v\right)_{n}-\left(\partial_{n} v_{t}\right)_{n}+\left(\partial_{n} Y_{t} \partial_{y} v\right)_{n}-\left(\partial_{n} v\right) \cdot \widehat{D}_{t} n .
$$

We finally find

$$
\int_{\Gamma}\left(\partial_{n} v_{t}\right)_{\tau}\left(v_{t}\right)_{\tau}=\int_{\Gamma}\left(v_{t}\right)_{n}\left(\partial_{n} v_{t}\right)_{n}+\int_{\Gamma} \pi_{t}^{1}
$$

with

$$
\begin{aligned}
\pi_{t}^{1}= & \bar{\pi}_{t}^{1}+\tilde{\pi}_{t}^{1}+\left(v_{t}\right)_{n}\left[\left(\widehat{D}_{t} \tau \cdot \nabla v\right)_{\tau}-\left(\partial_{\tau} Y_{t} \partial_{y} v\right)_{\tau}+\left(\partial_{\tau} v\right) \cdot \widehat{D}_{t} \tau\right. \\
& \left.+\left(\widehat{D}_{t} n \cdot \nabla v\right)_{n}-\left(\partial_{n} Y_{t} \partial_{y} v\right)_{n}+\left(\partial_{n} v\right) \cdot \widehat{D}_{t} n\right] .
\end{aligned}
$$

We have therefore recovered the boundary integral over $\left[p_{t}-2 \varepsilon\left(\partial_{n} v_{t}\right)_{n}\right] \cdot\left(v_{t}\right)_{n}$. The error term is an integral over $\pi_{t}^{1}$ with

$$
\pi_{t}^{1} \in \mathcal{P}\left(\bar{\partial}^{1} \nabla h, \bar{D}^{1} v, \bar{D}^{1} Y_{t}\right)
$$

We again want to identify, up to error terms, the boundary integrals on the left-hand side of (4.8) with the time derivative of an energy. We evaluate 


$$
\begin{aligned}
& p_{t}(x, h(x, t), t)-2 \varepsilon\left(\partial_{n} v_{t}\right)_{n} \\
& \stackrel{(2.3)}{=} \frac{\mathrm{d}}{\mathrm{d} t}\left[p(x, h(x, t), t)-2 \varepsilon\left(\partial_{n} v\right)_{n}\right]+2 \varepsilon\left[\left(\widehat{D}_{t} n \cdot \nabla v\right)_{n}-\left(\partial_{n} Y_{t} \partial_{y} v\right)_{n}+\partial_{n} v \cdot \widehat{D}_{t} n\right] \\
& \quad=-\nabla \cdot\left(\frac{\partial_{t} \nabla h}{\sqrt{1+|\nabla h|^{2}}}\right)+\varepsilon \sigma_{t}^{1}
\end{aligned}
$$

with

$$
\sigma_{t}^{1}:=2\left[\left(\widehat{D}_{t} n \cdot \nabla v\right)_{n}-\left(\partial_{n} Y_{t} \partial_{y} v\right)_{n}+\partial_{n} v \cdot \widehat{D}_{t} n\right] .
$$

The equation

$$
v_{t} \cdot n=\frac{\partial_{t}^{2} h}{\sqrt{1+|\nabla h|^{2}}}+\delta_{t}^{1}
$$

remains unchanged. We find for the boundary integral

$$
\begin{aligned}
\int_{\Gamma}\left[p_{t}-2 \varepsilon\left(\partial_{n} v_{t}\right)_{n}\right]\left(v_{t}\right)_{n}= & \int_{S}\left(-\nabla \cdot \frac{\partial_{t} \nabla h}{{\sqrt{1+|\nabla h|^{2}}}^{3}}+\varepsilon \sigma_{t}^{1}\right)\left(\partial_{t}^{2} h+\delta_{t}^{1} \sqrt{1+|\nabla h|^{2}}\right) \\
= & \partial_{t} \frac{1}{2} \int_{S} \frac{\left|\partial_{t} \nabla h\right|^{2}}{{\sqrt{1+|\nabla h|^{2}}}^{3}}-\int_{S} \varepsilon_{t}^{1}+\int_{S} \varepsilon \sigma_{t}^{1} \cdot\left(v_{t}\right)_{n} \sqrt{1+|\nabla h|^{2}} \\
& +\int_{S}\left(\frac{\partial_{t} \nabla h}{{\sqrt{1+|\nabla h|^{2}}}^{3}}\right) \cdot \nabla\left(\delta_{t}^{1} \sqrt{1+|\nabla h|^{2}}\right) .
\end{aligned}
$$

The energy estimate corresponding to (2.8) reads now

$$
\begin{aligned}
\partial_{t} E_{t}^{1}= & -\varepsilon \int_{\Omega_{t}}\left|\nabla v_{t}\right|^{2}+\int_{\Omega_{t}} f_{t}^{1} \cdot v_{t}+\int_{\Omega_{t}} g_{t}^{1} p_{t}+\int_{\Omega_{t}} \varepsilon F_{t}^{1} \cdot v_{t} \\
& +\int_{S} \varepsilon_{t}^{1}-\int_{S}\left(\frac{\partial_{t} \nabla h}{\sqrt{1+|\nabla h|^{2}}}\right) \cdot \nabla\left(\delta_{t}^{1} \sqrt{1+|\nabla h|^{2}}\right) \\
& -\int_{S} \varepsilon \sigma_{t}^{1} \cdot\left(v_{t}\right)_{n} \sqrt{1+|\nabla h|^{2}}+\varepsilon \int_{\Gamma} \pi_{t}^{1} .
\end{aligned}
$$

We sketch the analysis of these error terms. On level $k+1$ the energy

$$
\sup _{\tau} E_{t}^{k+1}(\tau)+\varepsilon \int_{0}^{T} \int_{\Omega_{t}}\left|\nabla v_{t}^{k+1}\right|^{2} \mathrm{~d} t
$$

controls the norms

$$
\begin{aligned}
& \sqrt{\varepsilon} \nabla\left(v_{t}^{k+1} \circ X\right) \in L^{2}\left(0, T ; L^{2}(R)\right), \\
& \left.\sqrt{\varepsilon} v_{t}^{k+1}\right|_{\Gamma} \circ X \in L^{2}\left(0, T ; H^{1 / 2}(S)\right) .
\end{aligned}
$$

The estimates for $v$ obtained from the vorticity equation provide bounds for

$$
\begin{aligned}
& \nabla\left(v_{t}^{k} \circ X\right) \in L^{\infty}\left(0, T ; H^{1 / 2}(R)\right), \\
& \nabla\left(\left.v_{t}^{k}\right|_{\Gamma} \circ X\right) \in L^{\infty}\left(0, T ; L^{2}(S)\right),
\end{aligned}
$$


as in the inviscid case (see below). By taking horizontal derivatives we conclude the corresponding spatial estimates. With these bounds we can control the highest order terms of $\int_{\Omega_{t}} \varepsilon F_{t}^{k+1} \cdot v_{t}^{k+1}$. The expression $F_{t}^{k+1}$ has in highest order the terms $\tilde{F}_{t}^{k+1}=P_{i j}\left(\bar{D}^{1} v, \bar{D}^{1} Y_{t}\right) \partial_{i} \partial_{j} v_{t}^{k}$. Therefore, after one integration by parts,

$$
\begin{aligned}
\int_{\Omega_{t}} \varepsilon \tilde{F}_{t}^{k+1} \cdot v_{t}^{k+1} & =\int_{\Omega_{t}} \varepsilon \partial_{i} \partial_{j} v_{t}^{k} P_{i j}\left(\bar{D}^{1} v, \bar{D}^{1} Y_{t}\right) \cdot v_{t}^{k+1} \\
& =-\int_{\Omega_{t}} \varepsilon \partial_{j} v_{t}^{k} \partial_{i}\left[P_{i j}\left(\bar{D}^{1} v, \bar{D}^{1} Y_{t}\right) \cdot v_{t}^{k+1}\right]+\int_{\Gamma_{t}} \varepsilon \partial_{j} v_{t}^{k} n_{i} P_{i j}\left(\bar{D}^{1} v, \bar{D}^{1} Y_{t}\right) \cdot v_{t}^{k+1} .
\end{aligned}
$$

These integrals are bounded by the norms of (4.10) and (4.11).

For the boundary integral containing $\sigma_{t}^{k+1}$ we use that for $k \geqslant 3$

$$
\sigma_{t}^{k+1} \in \mathcal{P}^{(1,0)}\left(\bar{\partial}^{k+1} \nabla h, \bar{D}^{k} \nabla v\right) .
$$

This implies sufficient regularity since $\sigma_{t}^{k+1}$ is multiplied with $\bar{\partial}^{k+2} h$; for purely temporal estimates we use that $\sqrt{\varepsilon} \partial_{t}^{k+2} h$, has the regularity of $\left.\sqrt{\varepsilon} v_{t}^{k+1} \circ X\right|_{S}$.

The other integral that did not appear in the inviscid case is the boundary integral over $\varepsilon \pi_{t}^{k+1}$. For $k \geqslant 3$ we have

$$
\pi_{t}^{k+1} \in \mathcal{P}^{(2,0)}\left(\bar{\partial}^{k+1} \nabla h, \bar{D}^{k+1} v, \bar{D}^{k+1} Y_{t}\right) .
$$

The energy estimate includes a bound for $\bar{\partial}^{k+1} \nabla h \in L^{\infty}\left(I ; L^{2}(S)\right)$ and therefore this integral is bounded, too.

\subsection{Regularity properties of $v$ and $p$}

It remains to show the regularity properties of $v$ and $p$. The most important observation is that the $H_{*}^{-1 / 2}(R)$ estimate for $\partial_{t}^{k+1} p$ still holds, at least in an $L^{2}$-sense in time.

Step 1. Lemma 6 remains valid. We multiply (4.6) with $-\Delta \omega$ and integrate by parts on the left-hand side. The boundary terms vanish due to our condition $\omega=0$ on upper and lower boundary. The right-hand side yields a negative term in the energy inequality. For higher regularity one takes $k=2 m$ tangential or vertical derivatives and repeats the procedure. For the boundary estimates one exploits once more the special boundary condition.

Step 2. Lemma 4 holds also for the Navier-Stokes approximation: the above bounds for $h$ and $\omega$ imply estimates for $v$ as in (3.5), and the traces of $\bar{D}^{k} \nabla v$ are bounded in $L^{\infty} L^{2}(S)$.

The proof for the velocity estimates is identical to that in the inviscid case. We only used the knowledge on $\operatorname{div} v$ and curl $v$ and the boundary values in order to conclude the regularity of $v$.

Step 3. The pressure satisfies a bound

$$
\|p \circ X\|_{L^{2}\left(0, T ; H^{k+1 / 2}(R)\right)}+\cdots+\|p \circ X\|_{H^{k}\left(0, T ; H^{1 / 2}(R)\right)} \leqslant C .
$$

This estimate differs from (3.20) in two respects: the $L^{\infty}$-norm in time is replaced by an $L^{2}$-norm, and the orders of differentiability are reduced by one. This change does not affect the analysis of error terms concerning the pressure.

The proof for the above pressure estimates is based on an equation for $\Delta p$, that remains unchanged, and the boundary values for $p$. Here we have with (4.4) a variation with respect to the inviscid case:

$$
\nabla_{x} \cdot\left(\frac{\nabla_{x} h}{\sqrt{1+\left|\nabla_{x} h\right|^{2}}}\right)+p=2 \varepsilon\left(\partial_{n} v\right) \cdot n \text {. }
$$

In order to analyze $k+1$-st derivatives of the pressure we have to assure that $k+1$-st derivatives of the right-hand side have boundary values in $L^{2}\left(I ; H^{-1}(S)\right)$. This follows from the equality

$$
\left(\partial_{n} v_{t}^{k+1}\right)_{n} \circ X=\left(\partial_{\tau} v_{t}^{k+1}\right)_{\tau} \circ X+\text { l.o.t. }
$$


where 1.o.t. denotes terms of lower order in $v$. In highest order the error term for $p_{t}^{k+1}$ is therefore

$$
2 \varepsilon \partial_{x}\left[{\sqrt{1+\left|\partial_{x} h\right|^{2}}}^{-1}\left(v_{t}^{k+1} \cdot \tau\right) \circ X\right]
$$

Using (4.10) we have the desired estimate.

In the derivation of the interior pressure estimate of Lemma 7 we have a technical change. In Eq. (3.23) appears an additional term containing $F_{0}$ on the right-hand side.

$$
\mathcal{L} u=\nabla \cdot B \nabla F_{0}+\operatorname{div} F_{1}+F_{2} .
$$

Here $B$ is a smooth matrix field and $F_{0}$ contains $\partial_{t}^{k} p$; we have regularity bounds for $F_{0} \in L^{2}\left(0, T ; H^{1 / 2}(R)\right)$. In the third step in the proof of Lemma 7 we have seen that this still allows an estimate for $\tilde{u} \in L^{2}\left(0, T ; L^{2}(R)\right)$. This suffices for the estimate

$$
\|p \circ X\|_{H^{k+1}\left(0, T ; H_{*}^{-1 / 2}(R)\right)} \leqslant C .
$$

On the regularity of formal time derivatives. We approximate the Euler equations with a Navier-Stokes system; this implies that we change the formal time derivatives of the initial values, since we must now calculate them using the Navier-Stokes equations. Up to functions of order $\varepsilon$ they coincide with the formal time derivatives of the Euler system. Choosing $\varepsilon$ small enough (depending on regular norms of the initial values) we have the formal time derivatives bounded as demanded in Assumption 5. For the sake of an approximate solution it is sufficient to demand a smallness of $\varepsilon$ depending on smooth norms of the initial values.

Unfortunately, the existence result for our Navier-Stokes system can not be quoted from existing literature, we nevertheless omit the proof. It follows the lines of proofs for the standard Navier-Stokes system. Note that with the a priori estimate the key ingredient is presented in the work at hand.

\section{Appendix. Facts on fractional Sobolev spaces}

In this appendix we collect the results on the fractional Sobolev spaces $H^{s}$ that are used in this work.

A result of Calderon concerns the complex interpolation ([16], p. 40):

$$
\left[H^{s_{0}}, H^{s_{1}}\right]_{\Theta}=H^{s}, \quad s=(1-\Theta) s_{0}+\Theta s_{1} .
$$

See [15], p. 204 for the corresponding result on bounded domains. The interpolation functor has the property that a bounded linear map $T: A_{j} \rightarrow B_{j}$ (for $j=0,1$ ) is bounded as a map ([16], p. 39)

$$
T:\left[A_{0}, A_{1}\right]_{\Theta} \rightarrow\left[B_{0}, B_{1}\right]_{\Theta} .
$$

For a smooth bounded domain $\Omega$ we have the trace theorem ([16], p. 212). For $s>1 / 2$ the trace is a bounded linear function

$$
\text { trace }: H^{s}(\Omega) \rightarrow H^{s-1 / 2}(\partial \Omega) .
$$

The corresponding result for $s=1 / 2$ does not hold. See [10], p. 55 for the opposite result

$$
H_{0}^{1 / 2}(\Omega)=H^{1 / 2}(\Omega) .
$$

Boundary values can be extended to the interior by a harmonic function. For $s \geqslant 1 / 2$ this extension is a linear and bounded map

$$
\mathcal{H}: H^{s-1 / 2}(\partial \Omega) \rightarrow H^{s}(\Omega) .
$$


See [10], p. 188 for this result. The same reference provides that the map

$$
\left.H^{s-3 / 2}(\partial \Omega) \ni \partial_{n} u\right|_{\Gamma} \mapsto u \in H^{s}(\Omega),
$$

where $u$ denotes the harmonic solution of the boundary value problem, is linear and bounded.

Note that we are working not on $\mathbb{R}^{N-1} \times \mathbb{R}_{+}$, but rather with a periodicity condition in horizontal directions. The above mentioned results remain valid; compare the remark on page 252 of [16] and the discussion on page 266 in [15].

\section{References}

[1] J.T. Beale, Large time regularity of viscous surface waves, Arch. Rat. Mech. Anal. 84 (1984) 307-352.

[2] K. Beyer, M. Günther, On the Cauchy problem for a capillary drop. I. Irrotational motion, Math. Methods Appl. Sci. 21 (12) (1998) $1149-1183$.

[3] X. Chen, A. Friedman, A bubble in ideal fluid with gravity, J. Differential Equations 81 (1989) 136-166.

[4] D. Christodoulou, H. Lindblad, On the motion of the free surface of a liquid, Comm. Pure Appl. Math. 53 (12) (2000) 1536-1602.

[5] D.G. Ebin, The equations of motion of a perfect fluid with free boundary are not well posed, Comm. Partial Differential Equations 12 (1987) 1175-1201.

[6] L.C. Evans, Partial Differential Equations, Grad. Stud. Math., vol. 19, Amer. Math. Soc., 1998.

[7] T. Iguchi, N. Tanaka, A. Tani, On the two-phase free boundary problem for two-dimensional water waves, Math. Ann. 309 (2) (1997) 199-223.

[8] T. Iguchi, N. Tanaka, A. Tani, On a free boundary problem for an incompressible ideal fluid in two space dimensions, Adv. Math. Sci. Appl. 9 (1) (1999) 415-472.

[9] T. Kato, G. Ponce, Well-posedness of the Euler and Navier-Stokes equations in Lebesgue spaces, Rev. Mat. Iberoamericana 2 (1986) 73-88.

[10] J.L. Lions, E. Magenes, Non-Homogeneous Boundary Value Problems and Applications, I, Grundlehren Math. Wiss., vol. 181, SpringerVerlag, 1972.

[11] M. Ogawa, A. Tani, Free boundary problem for an incompressible ideal fluid with surface tension, Math. Models Methods Appl. Sci. 12 (12) (2002) 1725-1740.

[12] N. Okazawa, The Euler equation on a bounded domain as a quasilinear evolution equation, Commun. Appl. Nonlinear Anal. 3 (3) (1996) $107-113$

[13] M. Renardy, An existence theorem for a free surface flow problem with open boundaries, Comm. Partial Differential Equations 17 (1992) $1387-1405$.

[14] B. Schweizer, A two-component flow with a viscous and an inviscid fluid, Comm. Partial Differential Equations 25 (2000) 887-901.

[15] H. Triebel, Theory of Function Spaces, Monographs Math., vol. 78, Birkhäuser, 1983.

[16] H. Triebel, Theory of Function Spaces II, Monographs Math., vol. 84, Birkhäuser, 1992.

[17] A. Wagner, On the Bernoulli free boundary problem with surface tension, in: I. Athanasopoulos (Ed.), Free boundary problems: theory and applications, in: CRC Res. Notes Math., vol. 409, Chapman \& Hall, 1999, pp. 246-251.

[18] S. Wu, Well-posedness in Sobolev spaces of the full water wave problem in 2-D, Invent. Math. 130 (1) (1997) 39-72.

[19] S. Wu, Well-posedness in Sobolev spaces of the full water wave problem in 3-D, J. Amer. Math. Soc. 12 (2) (1999) $445-495$. 